\title{
Beiträge zum Kreatininstoffwechsel.
}

\section{Von}

Dr. G. Lefmann, wissenschaftlichem Assistenten.

(Aus der medizinischen Poliklinik zu Heidelberg. Dir. Geh. Hofrat Fleiner.)

(Der Redaktion zugegangen am 17. September 1908.)

\section{Einleitung.}

Seit der Vereinfachung der quantitativen Kreatininbestimmung durch die Folinsche ${ }^{1}$ ) Methode sind eine Reihe von Arbeiten erschienen, die den Kreatininstoffwechsel beim Menschen und beim Tier zum Gegenstand haben. Auch bei Kranken sind bereits unter Nutzbarmachung dieser Methode Untersuchungen über die Kreatininausscheidung angestellt worden; nahezu allen diesen Untersuchungen, soweit sie am normalen Organismus ausgeführt wurden, liegt der Gedanke zugrunde, über den Ablauf des Kreatininstoffwechsels bei Muskelruhe und bei Muskelarbeit Aufschluß zu schaffen, und den Einfluß zu studieren, welchen der Wechsel im Gehalte der Nahrung an Stickstoff und Purinbasen auf den Kreatininhaushalt hat. Der Ausführung solcher Untersuchungen stehen namentlich zwei Momente hindernd entgegen: Das eine besteht in der Schwierigkeit einer sicheren quantitativen und verlustlosen Überführung des Harnkreatins in Kreatinin, in welcher Form das Kreatin dann bestimmt wird; die zweite Schwierigkeit in der großen Abhängigkeit der Kreatinin- und Kreatinausscheidung vom Stoffwechsel der Gewebe, denen nachgewiesenermaßen sowohl eine aufbauende als zerstörende Funktion zukommt. Wie Gottlieb und Stangassing er ${ }^{2}$ ) gezeigt haben, ist die Menge des im Körper gebildeten Kreatinins nicht allein durch synthetische, sondern

1) Folin, Diese Zeitschrift, Bd. XLI, S. 223.

2) R. Gottlieb u. R. Stangassinger, Diese Zeitschrift, Bd. LI, S. 1. - R. Stangassinger, Diese Zeitschrift, Bd. LV, S. 295. 
auch durch zerstörende Prozesse innerhalb der Organe bedingt und die Annahme, daß der endogene Kreatin- und Kreatininstoffwechsel unter gleichen Versuchsbedingungen stets der gleiche sei, ist Voraussetzung für die Beurteilung zahlreicher Versuche, beispielsweise der Fütterungsversuche. Eine absolute Gewähr hierfür sich zu verschaffen, ist unmöglich; nur indirekt erhält man eine solche dadurch, daß man genügend große Normalperioden den eigentlichen Versuchen vorausschickt, ebenso wie man dies bei $\mathrm{N}$ - und $\overline{\mathrm{U}}$-Bestimmungen längst gewohnt ist. Dadurch erhält man aber immer nur Übersichtsbilder über den Gesamtstoffwechsel und eine Kontrolle der einzelnen in Betracht kommenden Organe fehlt nach wie vor. Eine solche wäre aber z. B. für die Leber, die bei dem Kreatininstoffwechsel, wie neuerdings gezeigt wurde, eine große Rolle spielt, unbedingt wünschenswert, da man analog den Erfahrungen an anderen Organen annehmen muß, daß die Arbeitsleistung der Leber auf verschiedene Reize ganz verschieden ausfällt. Wollte man den Versuch machen, diese Vorgänge zu analysieren, so blieb nichts anderes übrig, als zunächst den normalen Ablauf des Kreatininstoffwechsels zu untersuchen und dann die etwaigen Veränderungen zu studieren, welche nach sicherer Schädigung eines bestimmten Organes des gleichen Individuums auftreten. Das ist natürlich nur im Tierexperiment möglich und die nächste Aufgabe war, für die zur Verwendung kommenden Tiere Normalwerte bei stickstoff- und purinkörperarmer Kost zu schaffen. Im Anschluß hieran wurde dann der Einfluß verschiedenartig zusammengesetzter Nahrung sowie derjenige von Kreatingaben unter verschiedenen Umständen untersucht, wie dies aus den nachfolgenden Versuchsergebnissen hervorgeht.

\section{Versuchsanordnung.}

Die Versuche wurden ausschließlich an Hündinnen ausgeführt, denen zur Erleichterung des Katheterisierens eine Scheidenspaltung gemacht worden war. Die Tiere befanden sich in Stoffwechselkäfigen. Die Untersuchungen des Urins wurden durchweg am Mischurine gemacht und fanden stets direkt im 
Anschluß an das Katheterisieren statt; zweimal täglich, um $12 \mathrm{Uhr}$ mittags und um $6 \mathrm{Uhr}$ abends, wurden die Tiere katheterisiert. Im Urine wurde dann der Stickstoff nach Kjeldahl, Kreatinin und Kreatin nach der Folinschen Methode bestimmt. Als Kolorimeter wurde das von R. Gottlieb und Stangassinger ${ }^{1}$ ) beschriebene Modell benutzt. $\left.{ }^{2}\right)$ Die Bestimmung des Kreatinins im Harn ging ohne weiteres vonstatten. Schwieriger gestaltete sich die Invertierung von Kreatin zu Kreatinin im Harn. Für reine Kreatinlösungen hatten Gottlieb und Stangassinger gefunden, daß eine quantitative Umsetzung von Kreatin in Kreatinin am besten bei 2,2\% Salzsäuregehalt vor sich gehe. Setzte man mehr Säure zu, so fand eine nachträgliche Zerstörung des gebildeten Kreatinins statt. Auf dieser Angabe fußend versuchte ich auch im Urine eine Umsetzung von Kreatin in Kreatinin; ich erhielt dabei jedoch fast durchweg geringere Werte für den Gesamtgehalt an Kreatinin plus Kreatin als für Kreatinin allein, trotz sorgfältiger Neutralisierung nach mehr(3) stündigem Erhitzen auf dem Wasserbade. Es sind eben für die Umsetzung von Kreatin in Kreatinin in reinen Lösungen ganz andere Bedingungen erforderlich als im Harn und ähnlichen Flüssigkeiten. Dieselben müssen für jeden Versuchsplan besonders ausfindig gemacht werden. Dies veranlaßte mich, ganz genau nach der von Folin gegebenen Vorschrift zu verfahren, da dieselbe, für Urinwerte ausgearbeitet, noch die meisten Chancen für richtige Resultate darbietet. Nach Folinscher Vorschrift sollen $10 \mathrm{ccm}$ Urin mit $5 \mathrm{ccm}$ Normalsalzsäure 3 Stunden auf dem Wasserbade erhitzt, darnach neutralisiert und nach den Vorschriften der Kreatininbestimmung weiter behandelt werden. Statt $10 \mathrm{ccm}$ Urin verwendete ich öfters $20 \mathrm{ccm}$ unter entsprechender Vermehrung der Salzsäure, um die gewünschte Konzentration wieder herzustellen. Bis zur Trockene wurde nie eingedampft, um die Salzsäurekonzentration nicht $z u$ verstärken. Auch den früheren Untersuchern bot die Umwandlung von Kreatin in Kreatinin stets Schwierigkeiten; es handelte sich

1) Diese Zeitschrift, Bd. LII, Heft 1 u. 2.

2) Das von mir benutzte Kolorimeter war genau nach dem Runneschen Modell von dem Mechaniker P. Stoë in Heidelberg konstruiert. 
dabei jedoch immer nur um relativ einfach zusammengesetzte und meist eiweißreiche Flüssigkeiten. So kamen für meine Untersuchungen die von Jaffé, ${ }^{1}$ ) Dorner, ${ }^{2}$ ) Baur und Barschall, ${ }^{3}$ ) Gottlieb und Stangassinger, ${ }^{4}$ ) Weber ${ }^{5}$ ) ausgearbeiteten Methoden nicht in Betracht. van Hoogenhuyze und Verploegh ${ }^{6}$ ) geben nur Kreatininwerte an in ihren Untersuchungen, desgleichen Froschbach. ${ }^{7}$ ) Neuerdings hat sich dann Dreib$\left.h_{o l} z^{8}\right)$ mit der Überführung von Kreatin in Kreatinin beschäftigt, ohne eine brauchbarere Methode als die Folinsche angeben zu können. Mell anby ${ }^{9}$ ) benützte ebenfalls die Folinsche Methode der Invertierung mit gutem Erfolge. Er erwähnt dabei als besonders beachtenswert bei der Ausführung, daß die nach dem Erhitzen auf dem Wasserbade zur Verwendung kommende Flüssigkeitsmenge nicht etwa durch Auswaschen vergrößert werden dürfe. Ich ging stets so vor, daß ich die Jaffésche Reaktion in dem gleichen Gefäße vornahm, in dem der Urin mit Salzsäure erhitzt und neutralisiert worden war; darnach setzte ich soviel Wasser $z u$, als dem Volumen der ursprünglichen Urinmenge - gewöhnlich $20 \mathrm{ccm}$ - entsprach. Nach dem Eintreten der Reaktion wurde die gesamte Flüssigkeitsmenge in einen Meßkolben von $500 \mathrm{ccm}$ Inhalt gespült und zur kolorimetrischen Untersuchung verwendet. - Um im Urine ebenso vollständig das Kreatin in Kreatinin zu verwandeln, als dies bei den Organextrakten möglich ist, wurde versucht, denselben mit Eiweiß zu versetzen, nachdem sich Klärungsversuche mit Plumb. acet. und Tierkohle als nicht anwendbar

1) Jaffé, Diese Zeitschrift, Bd. XLVIII, S. 430.

2) Dorner, Ebenda, Bd. LII, S. 227.

8) Baur u. Barschall, Arbeiten a. d. kaiserl. Gesundheitsamt, Bd. XXIV, S. 552.

4) R. Gottlieb und R. Stangassinger, l. c.

5) Weber, Arch. für exp. Pathol. u. Pharm., Bd. LVIII, S. 93.

-) van Hoogenhuyze u. Verploegh, Diese Zeitschrift, Bd. XLVI, S. 465 .

7) Froschbach, Arch. f. exp. Pathol. u. Pharm., Bd. LVIII, S. 113.

8) Dreibholz, Wolfgang, Zur Frage der Kreatininausscheidung im Hame. Inaug.-Diss., Greifswald 1908.

9) Mellanby, Journal of Physiology, Bd. XXXVI, S. 288. 
gezeigt hat. Einen Abschluß haben diese Versuche noch nicht gefunden, einmal da die Folinsche Methode zunächst als ausreichend genau erschien, und zweitens, um nur Werte zu erhalten, die nach der gleichen Methode erhalten wurden und daher gut unter einander vergleichbar sind.

\section{Die Ausscheidung von Kreatin und Kreatinin unter normalen Verhältnissen.}

Wie schon erwähnt, beschäftigten sich die früheren Untersucher hauptsächlich mit dem Kreatinin- und Kreatinstoffwechsel bei Muskelarbeit und Muskelruhe, und die Ergebnisse dieser Untersuchungen sind ziemlich vollständig von C. J. C. van Hoogenhuyze und $\mathrm{H}$. Verploegh ${ }^{1}$ ) zusammengestellt worden, sodaß sie hier nicht nochmals in toto referiert werden sollen. Die genannten Untersucher führten an sich selbst größere Versuchsreihen unter verschiedenen Bedingungen aus, die $\mathrm{zu}$ interessanten Resultaten insofern führten, als trotz wechselnder $\mathrm{Zu}$ sammensetzung der Kost, die durchschnittliche Kreatinin- und Kreatinausscheidung keine großen Unterschiede zeigen, und auch kürzer dauernde Unterernährung sowie eingeschaltete Arbeitstage trotz großer körperlicher Anstrengung das Bild nicht wesentlich veränderten. Anders war es bei der von den Verfassern beobachteten Hungerkünstlerin Flora Tosca, bei der in 14 tägiger Hungerperiode der Kreatiningehalt des Urines stark sank und nur an den Arbeitstagen wieder etwas anstieg. Diese Versuchsresultate decken sich mit den früheren Beobachtungen über die Kreatininausscheidung bei Hunger und Muskelarbeit im großen und ganzen. Die betreffenden Angaben von Meissner, ${ }^{2}$ ) Moitessier, ${ }^{3}$ ) Gregor 4 ) und Grocco,5) Oddi und Tarulli ${ }^{6}$ ) beziehen sich ebenfalls auf den Gesamtstoffwechsel und

1) $1 . \mathrm{c}$.

2) Meissner, Zeitschrift für rationelle Medizin, 3. Reihe, Bd. XXXI, S. 284, 1868.

3) Compt. rend. Soc. biolog., Bd. XLIII, S. 573.

4) Gregor, Diese Zeitschrift, Bd. LI, S. 98.

5) Grocco, Ann. di chim. e di pharm., Bd. IV, S. 211. S. 2, 1893.

-) Oddi u. Tarulli, Boll. dell' acad. med. di Roma, Bd. XIX, 
sind nicht unbestritten. (Vgl. die Untersuchungen von Voit, ${ }^{1}$ ) Zantl ${ }^{2}$ ) und Hoffmann. ${ }^{3}$ )) In einer neueren Untersuchung hat dann Weber ${ }^{4}$ ) mittels der Folinschen Methode und unter $\mathrm{Zu}$ grundelegung einer sehr sinnreichen Versuchsanordnung festgestellt, daß die Muskelarbeit beim hungernden Tiere Kreatininvermehrung im Harne hervorruft, beim gleichmäßig ernährten jedoch die Kreatininausscheidung verringert. Natürlich wurde für die Vermehrung des Gesamtkreatinins bei Muskelarbeit in erster Linie das Kreatin der Muskeln verantwortlich gemacht und der ruhende und arbeitende Muskel auf seinen Kreatingehalt untersucht. Auch hier sind die Angaben widersprechend; von den früheren Autoren fand Liebig ${ }^{5}$ ) den Kreatingehalt bei Muskelarbeit höher als bei Muskelruhe; desgleichen Sarokin, 6) der doppelt so viel Kreatin im arbeitenden Muskel fand als im ruhenden, und ebenso Sczelkow; ${ }^{7}$ ) Voit ${ }^{8}$ ) und Nawrocki ${ }^{9}$ ) fanden das umgekehrte Verhältnis. Monaris ${ }^{10}$ ) Angaben sind wegen der angewandten Technik nicht einwandsfrei. Nach Webers Untersuchungen am Herzen über den Kreatinstoffwechsel finden sich während der Arbeit erhöhte Abgaben von Kreatin und von Kreatinin ${ }^{11}$ ) an die Durchblutungs(Ringersche) Flüssigkeit; in gelähmten Muskeln fand Weber weniger Kreatin als in normalen und arbeitsfähigen; Mellanby ${ }^{12}$ ) fand im Muskel so gut wie kein Kreatinin und bei

1) Voit, Zeitschrift f. Biolog., Bd. IV, S. 87.

2) Zantl, Über die Ausscheidung von Kreatin und Kreatinin bei verschiedener Nahrung. Inaug.-Diss., München 1868.

8) Hoffmann, Arch. f. pathol. Anatomie, Bd. XLVIII, S. 358.

4) Weber, l. c., S. 107.

5) Li ebig, Annal. d. Chem. u. Pharm., 1847, Bd. LXII, S. 257.

6) Sarokin, Arch. f. pathol. Anatom., 1863, Bd. XXVIII, S. 544.

7) Scelkow, Zentralbl. f. d. med. Wissenschaften, 1866, Nr. 31.

8) Voit, 1. c., S. 91.

9) Nawrocki, Zentralbl. f. die med. Wissensch., 1866, N. 40.

10) Monari, Malys Jahresber., 1889, Bd. XIX, S. 296.

11) Weber bestimmte nur den Gesamtgehalt der Ringerschen Flüssigkeit an Kreatinin und Kreatin; ob seine Werte auf vorhandenes Kreatinin oder nur auf invertiertes Kreatin zu beziehen sind, gibt er nicht an.

12) Mellanby, 1. c., S. 457 und 58. 
selbst langer Arbeit keine Umwandlung von Kreatin zu Kreatinin, ebensowenig einen Anstieg der Kreatinmenge im Muskel bei der Arbeit und im überlebenden Muskel selbst nach drei Tagen noch keinen Anstieg des Kreatingehaltes. Alle diese Untersuchungen zeigen - ganz abgesehen von den Fütterungsversuchen mit Kreatin und Kreatinin und Nahrungsmitteln, die reichlich eine oder beide Substanzen enthielten -, daß der Kreatininstoffwechsel des Muskels allein, sowie der Gesamtkreatininstoffwechsel einen höchst komplizierten Vorgang darstellt, sodaß man wohl die Frage zu stellen berechtigt ist, ob bei gleicher Ernährung und unter gleichen Versuchsbedingungen im Harne konstante Werte gefunden werden. Es wurden zur Entscheidung dieser Frage und der Frage nach der Ausscheidung von Kreatinin und Kreatin nach Fleischextraktfütterung zunächst bei zwei Hündinnen, die seit 10 Tagen nur Milch und Brot als Nahrung erhalten hatten, im Urine Stickstoff, Kreatinin und Kreatin bestimmt. Vor Beginn der eigentlichen Versuchsperiode wurde ein Hungertag eingeschaltet. Die Tiere erhielten zweimal $100 \mathrm{~g}$ Brot (meist Weißbrötchen) und $500 \mathrm{ccm}$ Milch vorgesetzt, fraßen jedoch, wie das aus den Urinmengen schon ersichtlich ist, recht wechselnde Mengen. Wie erwähnt wurden die Tiere zweimal katheterisiert, um die Urinmengen gut abzugrenzen, um $12 \mathrm{Uhr}$ und um $6 \mathrm{Uhr}$. Tabelle I und Tabelle II gibt die erhaltenen Werte wieder.

Betrachten wir die Tabelle I zuriächst, so sehen wir mit steigender Diurese und mit zunehmendem $\mathrm{N}$ vermehrte Ausscheidung von Kreatinin und Kreatin; dabei sind allerdings die Kreatimnengen minimal und meñmais so gering, đảs sie sich überhaupt nicht nachweisen ließen. ${ }^{1}$ )

An den 5 ersten Versuchstagen betrug die Menge des ausgeschiedenen Kreatins nur Bruchteile des Kreatinins. An dem eingeschalteten Hungertage gehen mit fallendem Stickstoffwerte auch diejenigen für Kreatin und Kreatinin zurück; am 6. Tage dagegen nahm der zweite Tageswert für Kreatinin erheblich zu, um am 7. Tage auf etwa das Dreifache des Durchschnitts-

1) Das Kreatin wurde stets in Kreatininwerten in den Tabellen angegeben; eine Umrechnung fand des leichteren Vergleiches wegen nicht statt. 
Tabelle I.

Hündin $10 \mathrm{~kg}$. Foxterrier. Seit 10 Tagen nur Milch und Brot als Nahrung.

\begin{tabular}{|c|c|c|c|c|c|c|}
\hline V.T. & $\begin{array}{c}\text { Urin- } \\
\text { menge } \\
\text { von } \\
6-12 \\
12-6 \\
\text { Uhr } \\
\text { ccm }\end{array}$ & $\mathrm{N}$ & $\begin{array}{l}\text { Krea- } \\
\text { tinin }\end{array}$ & Kreatin & $\begin{array}{c}\text { Gesamt- } \\
\text { wert für } \\
\text { Kreatinin } \\
\text { und } \\
\text { Kreatin }\end{array}$ & Bemerkungen \\
\hline \multirow[t]{3}{*}{1.} & 80 & 1,802 & 0,02360 & 0,00400 & 0,02760 & \multirow{3}{*}{ Brot und Milch. } \\
\hline & 135 & 0,996 & 0,04549 & - & 0,04549 & \\
\hline & 215 & 2,798 & 0,06909 & 0,00400 & 0,07309 & \\
\hline \multirow[t]{3}{*}{2.} & 222 & 2,198 & 0,12650 & 0,00890 & 0,13340 & \multirow{3}{*}{ Brot und Milch. } \\
\hline & 168 & 1,256 & 0,06720 & 0,00840 & 0,07560 & \\
\hline & 390 & 3,454 & 0,19370 & 0,01730 & 0,21100 & \\
\hline \multirow[t]{3}{*}{3.} & 425 & 3,408 & 0,20734 & - & 0,20734 & \multirow{3}{*}{ Brot und Milch. } \\
\hline & 108 & 0,815 & 0,05146 & 0,03924 & 9,09070 & \\
\hline & 533 & 4,223 & 0,25880 & 0,03924 & 0,29804 & \\
\hline \multirow[t]{3}{*}{4.} & 280 & 2,602 & 0,19804 & - & 0,19804 & \multirow{3}{*}{ Brot und Milch. } \\
\hline & 42 & 0,786 & 0,04660 & 0,00540 & 0,05200 & \\
\hline & 322 & 3,388 & 0,24464 & 0,00540 & 0,25004 & \\
\hline \multirow[t]{3}{*}{5.} & 129 & 2,421 & 0,2089 & \multirow{3}{*}{-} & 0,2089 & \multirow{3}{*}{ Hunger. } \\
\hline & 5 & 0,099 & - & & 0,00720 & \\
\hline & 134 & 2,520 & 0,2089 & & 0,21610 & \\
\hline \multirow[t]{3}{*}{6.} & 76 & 1,721 & 0,18964 & 0,01556 & 0,20520 & \multirow{3}{*}{$\begin{array}{l}\text { Hunger. Um } 1 \text { Uhr } 5 \mathrm{~g} \\
\text { Liebigs Fleischextrakt } \\
\text { und } 200 \mathrm{ccm} \text { Wasser. }\end{array}$} \\
\hline & 46 & 0,625 & 0,09315 & 0,01328 & 0,10644 & \\
\hline & 122 & 2,346 & 0,28279 & 0,02884 & 0,31164 & \\
\hline \multirow[t]{3}{*}{7.} & 175 & 2,191 & ৩,35350 & 0,11900 & 0,47250 & \multirow{3}{*}{$\begin{array}{l}\text { Un } 5 \text { Uhr am Aberrd romter } \\
5 \mathrm{~g} \text { Liebigs Fleischextr. } \\
\text { und } 200 \mathrm{ccm} \text { Wasser. }\end{array}$} \\
\hline & 107 & 0,886 & 0,18390 & 0,02796 & 0,21186 & \\
\hline & 282 & 3,077 & 0,53740 & 0,14696 & 0,68436 & \\
\hline
\end{tabular}

wertes zu steigen, während der Wert für Kreatin am gleichen Tage ebenfalls stark steigt. - Gleiche Verhältnisse finden wir in Tabelle II. Auch hier finden wir zunächst bei steigender Diurese und Vermehrung des ausgeschiedenen Gesamt- $\mathrm{N}$ einen Anstieg von Kreatinin - nicht von Kreatin - am zweiten Tage, während sonst vom 1 . Versuchstage an die Werte ziemlich gleich blieben. Auch hier betrug die gefundene Kreatin- 
Tabelle II.

Foxterrier $5 \mathrm{~kg}$. Seit 10 Tagen mit Milch und Brot genährt. Vor Beginn der Bestimmungen ein Hungertag.

\begin{tabular}{|c|c|c|c|c|c|c|}
\hline V. T. & $\begin{array}{c}\text { Urin- } \\
\text { menge } \\
\text { von } \\
6-12 ; \\
12-6 \\
\text { Uhr } \\
\mathrm{ccm}\end{array}$ & $\mathrm{N}$ & $\begin{array}{c}\text { Krea- } \\
\text { tinin }\end{array}$ & Kreatin & $\begin{array}{l}\text { Gesamt- } \\
\text { wert für } \\
\text { Kreatinin } \\
\text { und } \\
\text { Kreatin }\end{array}$ & Bemerkungen \\
\hline \multirow[t]{3}{*}{1.} & 55 & 1,19547 & 0,01740 & $|0,00443|$ & 0,02183 & \multirow{3}{*}{ Milch und Brot. } \\
\hline & 28 & 0,42473 & 0,03544 & 0,00142 & 0,03686 & \\
\hline & 83 & 1,62020 & 0,05284 & $0,00 \tilde{85}$ & 0,05896 & \\
\hline \multirow[t]{3}{*}{2.} & 212 & 2,11928 & 0,11832 & 0,00886 & 0,12718 & \multirow{3}{*}{ Milch und Brot. } \\
\hline & 92 & 0,06691 & 0,03841 & - & 0,03841 & \\
\hline & 304 & 2,18619 & 0,15673 & 0,00886 & 0,16559 & \\
\hline \multirow[t]{3}{*}{3.} & 150 & 1,24046 & 0,06750 & 0,00076 & 0,06826 & \multirow{3}{*}{ Milch und Brot. } \\
\hline & 67 & 0,49442 & 0,02185 & 0,00863 & 0,03048 & \\
\hline & 217 & 1,73488 & 0,08935 & 0,00939 & 0,09874 & \\
\hline \multirow[t]{3}{*}{4.} & 25 & 0,45335 & 0,04262 & $|0,00446|$ & 0,04708 & \multirow{3}{*}{ Milch und Brot. } \\
\hline & 25 & 0,60021 & 0,02700 & 0,00932 & 0,03632 & \\
\hline & 50 & 1,05356 & 0,06962 & $\mid 0,01378$ & 0,08340 & \\
\hline \multirow[t]{3}{*}{5.} & 30 & 0,63002 & $0,05 \check{340}$ & $0,00660 \mid$ & 0,06000 & \multirow{3}{*}{ Hunger. } \\
\hline & 10 & 0,18447 & 0,01556 & 0,00348 & 0,01904 & \\
\hline & 40 & 0,81449 & 0,06896 & 0,01008 & 0,07904 & \\
\hline \multirow[t]{3}{*}{6.} & 24 & $0,73255 \mid$ & 0,07068 & 0,00866 & 0,07934 & \multirow{3}{*}{$\begin{array}{c}\text { Hunger. Um } 1 \text { Uhr } 5 \mathrm{~g} \\
\text { Liebig s Fleischextrakt } \\
\text { in } 200 \mathrm{ccm} \text { Wasser. }\end{array}$} \\
\hline & 146 & 0,07021 & 0,13751 & 0,11411 & 0,25162 & \\
\hline & 170 & 0.80276 & 0.20819 & 0.12277 & 0.33096 & \\
\hline \multirow[t]{3}{*}{7.} & 194 & 1,67428 & 0.28571 & 0,16326 & 0,44897 & \multirow{3}{*}{$\begin{array}{l}\text { Um } 6 \text { Uhr am } 6 . \text { Tage } 5 \mathrm{~g} . \\
\text { Liebig und } 200 \mathrm{ccm} \\
\text { Wasser. Um } 1 / 81 \mathrm{Uhr} \\
0,03 \text { Morph. subkutan. }\end{array}$} \\
\hline & 130 & 1,05498 & 1,21927 & 0,09983 & 0,31910 & \\
\hline & 324 & 2,72926 & 0,50498 & 0,26309 & 0,76807 & \\
\hline 8. & 146 & 1,47096 & 0,12716 & 0,10037 & 0,22743 & \\
\hline
\end{tabular}

menge nur Bruchteile, $1 / 10$ bis $1 / \bar{s}$ etwa der ermittelten Kreatininmenge. Nur an den drei letzten Tagen änderte sich das Bild. Mit stark vermehrter Kreatininausscheidung ging eine nahezu gleichgroße Kreatinausfuhr einher. Der Stickstoffgehalt 
des Urins nahm zwar zu, aber bei weitem nicht in gleichem Maße wie die Kreatinin- und die Kreatinmenge, was der bereits von Folin gemachten Beobachtung, daß die Kreatin- und Kreatininausscheidung unabhängig von dem Stickstoffwechsel sei, entspricht. Eine größere Morphingabe am 7. Tage, um Muskelruhe herbeizuführen, hat auf die Kreatin- und Kreatininausscheidung keinen Einfluß. In beiden Versuchen finden wir demnach sofort nach Darreichung von $5 \mathrm{~g}$ Liebigs Fleischextrakt erhebliche Vermehrung des ausgeschiedenen Kreatinins und Kreatins und zwar in Tabelle I vom 5 . auf den 6. Versuchstag um $0,081 \mathrm{~g}$; vom 6 . auf den 7 . Versuchstag um 0,25 g. In Tabelle II vom 5. auf den 6. Tag um $0,14 \mathrm{~g}$, vom 6. auf den 7. Tag um 0,30 g, jeweils unter gleichzeitiger Vermehrung des Gesamtstickstoffs. Die Kreatinmenge stieg in Tabelle I zunächst kaum; dann um $0,12 \mathrm{~g}$, in Tabelle II um $0,12 \mathrm{~g}$ vom 5. auf den 6. Tag, und um 0,14 g vom 6. auf den 7. Tag. Aus diesen Versuchen geht hervor, daß bei der Fütterung kleiner Mengen von Fleischextrakt die Kreatinin- und Kreatinausscheidung vermehrt wird. Diese Vermehrung kommt natürlich durch vergrößerte Zufuhr sowohl von Kreatin als von Kreatinin zustande. Die Menge des im Fleischextrakt vorhandenen Kreatinins sowohl wie Kreatins ist dabei sehr wechselnd, wie schon von $W$ eber betont ward. Bei dem von mir verwendeten Präparate überstieg die in demselben gefundene Kreatininmenge die Kreatinmenge weitaus, wie dies auch in den ausgeschiedenen Mengen der Tabellen I und II zum Ausdruck kommt. Der Kreatiningehalt des verfütterten Fleischextraktes betrug für $5 \mathbf{g}$ $0,165 \mathrm{~g}$, die Vergröberung der Kreatīninausfuhrmenge ist also durch die vermehrte Zufuhr vollauf erklärt, eine Umwandlung von Kreatin in Kreatinin ist nicht zu konstatieren, da sonst die Kreatininwerte noch bedeutend größere hätten werden müssen. Dieses Ergebnis steht in gutem Einklang mit der Behauptung Folins ${ }^{1}$ ) und af Klerckers, ${ }^{2}$ ) die überhaupt keine (Folin) oder so gut wie keine Vermehrung des Kreatinins, sondern nur des Kreatins fanden; dagegen fand Meissner, daß alles ein-

1) Folin, Festschrift für Hammarsten, 1906.

2) af Klercker, Biochem. Zeitschrift, Bd. III (S. 45-87), S. 63. 
geführte Kreatin im Harne als Kreatinin wieder erscheine; auch Weber beobachtete nach Verfütterung von Fleischextrakt eine Zunahme von Kreatinin, die jedoch wie die von Froschbach an seinem Basedowpatienten gefundene auf Rechnung der eingeführten Kreatininmenge zu setzen ist. Im Hühnermuskel fand Mellanby nach Kreatinfütterung keine Vermehrung von Kreatinin oder Kreatin. Es kann also auch nach unseren Erfahrungen keinem Zweifel unterliegen, daß der Organismus des Hundes nicht imstande ist, in Form von Liebigs Fleischextrakt verfüttertes Kreatin in Kreatinin umzuwandeln und als solches auszuscheiden.

\section{Die Ausscheidung von Kreatinin und Kreatin bei stickstoff- reicher Nahrung.}

Die Frage nach der Herkunft des Kreatinins und Kreatins ist noch immer nicht endgültig entschieden. Nach Folin ist die Menge des zugeführten Stickstoffs für die Ausfuhrgröße von Kreatinin und Kreatin belanglos; im Gegensatz zu den früheren Annahmen, bei denen die mit der Nahrung eingeführte Kreatinmenge die Hauptbedeutung für die Vermehrung der Kreatininausscheidung hatte, stehen Burian ${ }^{1}$ ) und $\mathrm{Jaff}^{2}$ ) auf dem Standpunkte, daß die Nucleinsubstanzen für die Bildung von Kreatinin und Kreatin nicht in Betracht kämen, und Dorner ${ }^{3}$ ) suchte diese Auffassung, die auch Magnus-Levy ${ }^{4}$ ) vertritt, dadurch zu stützen, daß er größere Mengen von Thymus einem Hunde verfütterte. Eine Vermehrung der Kreatininausfuhr fand darauf nicht statt und auch Froschbach ${ }^{b}$ ) konnte bei seinem Basedowkranken eine solche nicht finden. Ohne auf die Frage näher eingehen zu wollen, inwieweit der Organismus die Fähigkeit hat, aus den Vorstufen des Kreatins

1) Burian, Diese Zeitschrift, Bd. XLIII, S. 546.

2) Jaffé, Diese Zeitschrift, Bd. XLVIII, S. 463.

$\left.{ }^{8}\right)$ Dorner, Diese Zeitschrift, Bd. LII, S. 227.

4) Magnus Levy, siehe von Noorden, Pathol. d. Stoffwechsels, Bd. I, S. 136 u. 137, 1906.

5) Froschbach, Arch. f. experim. Pathol. u. Pharm., Bd. LVIII (S. 112-140), S. $134 \mathrm{ff}$. 
solches zu bilden - Jaffé $e^{1}$ ) hat hierüber ausgedehnte Versuche angestellt, ebenso Czernecki ${ }^{2}$ ) und Mellanby ${ }^{8}$ ) 一, wurde in einer Versuchsreihe, welche in Tabelle III wiedergegeben ist, der Einfluß verschiedener, an Nucleinen und Purinbasen reicher Nahrung auf die Kreatinin- und Kreatinausscheidung zu ermitteln gesucht. Diese Versuche wurden an der gleichen Hündin angestellt, deren Kreatinin- und Kreatinausscheidungswerte bei Milch- und Brotdiät in Tabelle I wiedergegeben sind. Der erste Blick auf Tabelle III zeigt schon, da $\beta$ an keinem Versuchstage die Kreatininausscheidung eine solche Höhe erreichte, wie nach Verfütterung von Fleischextrakt, obwohl die N-Ausscheidung fast durchweg ungleich höher war als an den betreffenden Tagen. Aber auch der Vergleich der Kreatininwerte der Tabelle III mit denjenigen der Milch- und Brottage in Tabelle I lehrt, daß eine Zunahme der Kreatininausscheidung absolut nicht stattfand, während die Kreatinwerte der Tabelle III die der Tabelle I um das Vielfache übersteigen. Diese vermehrte Ausscheidung von Kreatin ist natürlich auf die gesteigerte Zufuhr zurückzuführen und läßt in gewissen Grenzen geradezu einen direkten Schluß auf den Kreatingehalt der einzelnen eingeführten Nahrungsstoffe zu. Wenn trotzdem keine Vermehrung der Kreatininausfuhr stattfand, so ist das nur darauf zurückzuführen, daß der Hundeorganismus nicht imstande ist, aus dem mit der Nahrung eingeführten Kreatin Kreatinin zu bilden oder daß, wenn dies geschah, wieder eine Zerstörung des gebildeten Kreatinins im Organismus zustande kam. Von den verschiedenen in Tabelle III verfütterten Organen wurden früher bereits Muskeffleisch und Thymus zur Fütterung verwendet. Af Klercker ${ }^{4}$ ) untersuchte an sich selbst den Einfluß von großen Fleischmengen und fand dabei «keine merkbare Vermehrung des Harnkreatinins", ein Befund, der mit dem aus Tabelle III ersichtlichen völlig übereinstimmt. Dorner ${ }^{5}$ )

1) J affé, Diese Zeitschrift, Bd. XLVIII, S. 430.

2) Czernecki, Diese Zeitschrift, Bd. XEIV, S. 294.

3) Mellanby, l. c. S. 466.

${ }^{4)}$ af Klercker; l. c.

s) Dorner, l. c. 
Tabelle III.

Foxterrier $10 \mathrm{~kg}$. Mit Milch und Brot genährt. Vor Beginn der Versuche 1 Hungertag.

\begin{tabular}{|c|c|c|c|c|c|c|}
\hline V. T. & $\begin{array}{l}\text { Urin- } \\
\text { menge } \\
\text { von } \\
6-12 ; \\
12-6 \\
\text { Uhr } \\
\mathrm{ccm}\end{array}$ & $\mathrm{N}$ & $\begin{array}{l}\text { Krea- } \\
\text { tinin }\end{array}$ & Kreatin & $\begin{array}{c}\text { Gesamt- } \\
\text { wert für } \\
\text { Krea- } \\
\text { tinin } \\
\text { und } \\
\text { Kreatin }\end{array}$ & Bemerkungen \\
\hline \multirow[t]{3}{*}{1.} & 220 & 2,33142 & 0,01620 & - & 0,01620 & \multirow{3}{*}{ Milch und Brot. } \\
\hline & 150 & 0,71605 & 0,05376 & - & 0,05376 & \\
\hline & 370 & 3,04747 & 0,06996 & - & 0,06996 & \\
\hline \multirow[t]{3}{*}{2.} & 506 & 4,65466 & 0,20912 & 0,27836 & 0,48748 & \multirow{3}{*}{$\begin{array}{l}\text { Um } 6 \text { Uhr abends und } 8 \text { Uhr } \\
\text { morgens je } 220 \mathrm{~g} \text { Rind- } \\
\text { fleisch in } 300 \mathrm{ccm} \text { Wasser } \\
\text { gekocht. }\end{array}$} \\
\hline & 167 & 2,07738 & 0,04659 & 0,08049 & 0,12708 & \\
\hline & 673 & 6,73204 & 0,25571 & 0,35885 & 0,61456 & \\
\hline \multirow[t]{3}{*}{3.} & 320 & 5,63400 & 0,18001 & 0,46799 & 0,64800 & \multirow{3}{*}{ Wie am 2. Tage. } \\
\hline & 290 & 3,71500 & 0,09248 & 0,39689 & 0,48937 & \\
\hline & 610 & 9,34900 & 0,27249 & 0,86488 & 1,13737 & \\
\hline \multirow[t]{3}{*}{4.} & 465 & 5,00100 & 0,14486 & 0,08554 & 0,23040 & \multirow{3}{*}{$\begin{array}{l}\text { Um } 6 \text { Uhr abends und } 8 \text { Uhr } \\
\text { morgens je } 220 \mathrm{~g} \text { Rinder- } \\
\text { milz in } 300 \mathrm{ccm} \text { Wasser } \\
\text { gekocht. }\end{array}$} \\
\hline & 50 & 1,40400 & 0,04601 & 二 & 0,04601 & \\
\hline & 515 & 6,40500 & 0,19095 & 0,08554 & 0,27641 & \\
\hline \multirow[t]{3}{*}{5.} & 550 & 3,72420 & 0,13338 & 0,05695 & 0,19033 & \multirow{3}{*}{$\begin{array}{l}\text { Je } 200 \mathrm{~g} \text { Rindermilz wie } \\
\text { am 4. Tage. }\end{array}$} \\
\hline & 127 & 1,26080 & 0,07913 & 0,02214 & 0,10127 & \\
\hline & 677 & 4,98500 & 0,21251 & 0,07909 & $\mid 0,29160$ & \\
\hline \multirow[t]{3}{*}{6.} & 390 & 4,63080 & 0,20250 & 0,14850 & $|0,35100|$ & \multirow{3}{*}{$\begin{array}{l}\text { Je } 200 \mathrm{~g} \text { Rinderleber in } \\
300 \mathrm{ccm} \text { Wasser gekocht. }\end{array}$} \\
\hline & 175 & 2,15180 & 0,06197 & 0,02243 & 0,08440 & \\
\hline & 565 & 6,78260 & 0,26447 & 0,17093 & 0,43540 & \\
\hline \multirow[t]{3}{*}{7.} & 280 & 3,23380 & 0,10401 & 0,11235 & 0,21636 & \multirow{3}{*}{$\begin{array}{l}\text { Je } 200 \mathrm{~g} \text { Rinderleber in } \\
\text { 3M crm Wsssar gaknabt. }\end{array}$} \\
\hline & 63 & 1,58640 & 0,04399 & 0,01400 & 0,05799 & \\
\hline & 343 & 4.82020 & 0,14800 & 0,12635 & 0,27435 & \\
\hline 8. & 245 & 2,40650 & 0,16267 & - & $0,16267 \mid$ & Milch und Brot. \\
\hline 9. & 550 & 2,46150 & 0,12375 & 0,03312 & 0,15687 & $\begin{array}{l}\text { Je } 200 \mathrm{~g} \text { Thymus in } 300 \mathrm{ccm} \\
\text { Wasser gekocht. }\end{array}$ \\
\hline \multirow[t]{3}{*}{10.} & 335 & 3,01371 & 0,13301 & 0,00403 & $0,13704 \mid$ & \multirow{3}{*}{$\begin{array}{l}\text { Um } 6 \text { Uhr am } 9 . \text { Tage } 200 \mathrm{~g} \\
\text { Thymus in } 300 \text { ccmWasser. } \\
\text { Milch und Brot. }\end{array}$} \\
\hline & 114 & 1,32621 & 0,06891 & 0,01209 & 0,08100 & \\
\hline & 449 & 4,37992 & 0,20192 & 0,01612 & 0,21804 & \\
\hline \multirow[t]{3}{*}{11.} & 198 & 1,64011 & 0,10150 & 0,00834 & $|0,10984|$ & \multirow{3}{*}{ Milch und Brot. } \\
\hline & 140 & 0,95693 & 0,07460 & 一 & 0,07460 & \\
\hline & 338 & 2,59704 & 0,17610 & 0,00834 & $|0,18444|$ & \\
\hline
\end{tabular}


fand bei seinen Hunden nach Thymusverfütterung keine $\mathrm{Zu}$ nahme des Kreatinins und Froschbach, wie erwähnt, ebensowenig, was ebenfalls mit unseren Erfahrungen übereinstimmt. Aber noch andere Schlüsse lassen sich aus der Tabelle III ziehen. Abgesehen von der deutlich erkennbaren Unabhängigkeit der Kreatininausscheidung von Eiweißsubstanzen muß man wohl annehmen, daß nach Darreichung von Thymus, Leber, Milz und Muskeln die Ausscheidung anderer harnfähiger Substanzen, der Harnsäure und des Harnstoffs beispielsweise, eine vollständig andere ist, als nach Verabreichung von Milch und Brot. Das kann man wohl auch ohne genaue Bestimmung ohne weiteres behaupten; damit ist aber zugleich der Beweis erbracht, daß, da in Tabelle I und Tabelle III die Kreatininwerte gleichgeblieben sind, die Ausscheidung von Kreatinin von der ausgeschiedenen Harnsäure- und Harnstoffmenge absolut nnabhängig ist, mit anderen Worten, daß es sich bei der Bildung von Kreatin und Kreatinin um völlig andere Prozesse als bei der Harnstoff- und Harnsäurebildung handelt.

\section{Die Kreatinin- und Kreatinausscheidung nach parenteral zugeführtem Kreatin.}

Neben den zahlreichen Untersuchungen darüber, ob und inwieweit in den Körper eingeführter Fleischextrakt oder reines Kreatin die Kreatininausscheidung verändert, ist die Frage nach dem Schicksal des eingeführten Kreatins fast völlig in den Hintergrund getreten. Die Untersuchungen Meissners, ${ }^{1}$ ) nach denen subkutan injiciertes Kreatin sowohl wie Kreatinin vollständig wieder ausgeschieden werden, können wegen der Unvollkommenheit der Methodik als nicht mehr völlig beweisende angesehen werden. Hoogenhuyze und Verploegh ${ }^{2}$ ) geben in ihrem Selbstversuche nur ihre Erfahrungen nach Kreatininaufnahme wieder; Voit, ${ }^{3}$ ) der Hunden Kreatin verfütterte, fand nicht alles Kreatin im. Urine wieder. Nur af Klercker ${ }^{4}$ )

1) Meissner, l. c., S. 283.

2) Hoogenhuyze und Verploegh, 1. c. S. 437/38.

s) Voit, Zeitschrift f. Biologie, Bd. IV, 1868.

) af Klercker, l. c. S. $63-82$. 
bespricht die Kreatinausscheidung nach Fleischextraktaufnahme und nach Kreatingaben per os. Af Klercker fand stets nach Fleischextraktaufnahme Zunahme des Harnkreatins, nach Einnahme von selbst $2 \mathrm{~g}$ wasserfreiem Kreatin «keine bemerkbaren Mengen» im Harne. Er knüpfte an diesen Befund die Betrachtung, daß entweder das Liebigsche Fleischextrakt. Substanzen enthalte, aus denen der Organismus Kreatin zu bilden imstande sei, oder aber daß das Ausbleiben einer Vermehrung des Harnkreatins nach Kreatinaufnahme mit der Größe des Eiweißumsatzes in Zusammenhang insofern stände, als bei großem Eiweißumsatz eher Kreatin ausgeschieden würde als bei geringerem. Diese letztere Annahme wird durch einen Vergleichsversuch gestützt. Auch Folin ${ }^{1}$ ) teilt auf Grund seiner Erfahrung diese Anschauung und kommt zu dem Schlusse, daß das Kreatin ein Nahrungsmittel für den Gewebsstoffwechsel sei, das um so eher retiniert wird, je geringer der Vorrat an kreatinbildendem Material sei. Über den Ort dieser Retention ist jedoch nichts bekannt, man muß indessen wohl in erster Linie hierbei an die Muskulatur denken. Nun hat aber Mellanby nachgewiesen, daß, nachdem die Muskeln einen gewissen Gehalt an Kreatin erreicht haben, durch Kreatinfütterung der Tiere keine Vermehrung des Muskelkreatins für gewöhnlich erzielt wird und daß eine solche nur bei ganz jungen Tieren möglich ist. Dieser Befund spricht direkt gegen eine Retention in den Muskeln, außerdem liegt der Gedanke nahe, daß beim hungernden Tiere dann eher eine Retention zu erwarten sei, als beim gleichmäßig ernährten. Um gerade diese letztere Möglichkeit zu entscheiden, wurde eine Anzahl Versuche unter verschiedenen Bedingungen angestellt, bei denen nur reines (Kahlbaumsches) Kreatin verwendet wurde. Das Kreatin wurde stets parenteral zugeführt; es geschah dies aus verschiedenen Gründen. Einmal sollte - namentlich bei der intravenösen Injektion - eine «Überschwemmung » des Organismus mit Kreatin erzielt werden; dann ist die Sicherheit der vollständigen Einverleibung einer gewählten Menge durch subkutane oder

1) Festschrift für Hammarsten, Upsala und Wiesbaden 1906. 
intravenöse Applikation stets eine größere als bei der Fütterung, wenn man nicht gerade die Schlundsonde verwendet, wobei man stets auch noch verschiedenen Zufälligkeiten (Erbrechen namentlich) ausgesetzt ist. Schließlich sollte das eingeführte Kreatin dem Einfluß der Verdauungssäfte entzogen bleiben. Die Versuche wurden an vier verschiedenen Tieren ausgeführt; das erste derselben erhielt $0,5 \mathrm{~g}$ Kreatin einmal subkutan und einmal intravenös; das zweite $0,5 \mathrm{~g}$ intravenös. Das dritte Tier erhielt wieder einmal subkutan und einmal intravenös Kreatin, das vierte nur subkutan. Während der Versuche wurden die Tiere, welche tagelang vorher nur mit Milch und Brot ernährt worden waren, entweder in gleicher Weise weiter gefüttert oder sie hungerten oder sie bekamen schon vor Beginn des Versuchs kein Futter mehr. Das zur Verwendung kommende Kreatin wurde in physiologischer Kochsalzlösung gelöst und bei der intravenösen Applikation in leichter Äthernarkose aus einer Burette in die freigelegte Vena jugularis injiziert. Tabelle IV gibt die Resultate des Versuchs wieder, die nach

Tabelle IV.

Hündin $10 \mathrm{~kg}$. Mit Brot und Milch ernährt. Vor Beginn des Versuches kein Hungertag.

\begin{tabular}{|c|c|c|c|c|c|c|}
\hline V. T. & $\begin{array}{c}\text { Urin- } \\
\text { menge } \\
6-12 ; \\
12-6 \mathrm{Uhr} \\
\mathrm{ccm}\end{array}$ & $\mathbf{N}$ & Kreatinin & Kreatin & $\begin{array}{l}\text { Gesamt- } \\
\text { wert für } \\
\text { Kreatinin } \\
\text { und } \\
\text { Kreatin }\end{array}$ & Bemerkungen \\
\hline \multirow[t]{2}{*}{1.} & $\begin{array}{r}375 \\
19\end{array}$ & $\begin{array}{l}2,781 \\
0,897\end{array}$ & $\begin{array}{l}0,24000 \\
0,05540\end{array}$ & $\begin{array}{l}0,00370 \\
0,06180\end{array}$ & $\begin{array}{l}0,24370 \\
0,11000\end{array}$ & \multirow[t]{2}{*}{ Honngor 1) } \\
\hline & 393 & 3,068 & 0,29540 & 0,06490 & 0,36030 & \\
\hline \multirow[t]{2}{*}{2.} & $\begin{array}{l}70 \\
25\end{array}$ & $\begin{array}{c}1,849 \\
-\end{array}$ & $\begin{array}{l}0,18410 \\
0,10120\end{array}$ & $\begin{array}{c}0,07350 \\
-\end{array}$ & $\begin{array}{l}0,25760 \\
0,10120\end{array}$ & \multirow[t]{2}{*}{ Hunger } \\
\hline & 95 & 1,849 & 0,28530 & 0,07350 & 0,35880 & \\
\hline \multirow[t]{2}{*}{3.} & $\begin{array}{r}124 \\
70\end{array}$ & $\begin{array}{l}3,259 \\
1,089\end{array}$ & $\begin{array}{l}0,20460 \\
0,06020\end{array}$ & $\begin{array}{l}0,06696 \\
0,00350\end{array}$ & $\begin{array}{l}0,27156 \\
0,06370\end{array}$ & \multirow{2}{*}{$\begin{array}{c}\text { Milch und } \\
\text { Brot }\end{array}$} \\
\hline & 194 & 4,348 & 0,26480 & 0,07046 & 0,33526 & \\
\hline
\end{tabular}

1) Um 1 Uhr erhält das Tier $0,5 \mathrm{~g}$ Kreatin subkutan in $50 \mathrm{ccm}$ physiol. Kochsalzlösung. 
subkutaner Injektion von $0,5 \mathrm{~g}$ Kreatin bei einer Hündin erhalten wurden, die während des Versuchs (2 Tage) hungerte, bis zum Beginne desselben jedoch mit Milch und Brot ernährt worden war. Wenn man die Werte der Kreatinrubrik untereinander vergleicht, so nimmt man zunächst keine Vermehrung des ausgeschiedenen Kreatins wahr. Man kann aber trotzdem mit Sicherheit behaupten, daß der zweite Wert des ersten Tages die Norm übersteigt. Einmal ist er ungleich größer, als der erste Wert desselben Tages, was zwar vorkommen kann, aber auffällig ist, dann aber ist der Kreatinwert größer als der Kreatininwert. Das kommt bei dieser Ernährung resp. im Hunger unter normalen Verhältnissen nicht vor. Die Werte des 2. und 3. Versuchstages sind unverändert. Es fand also zweifellos auf die Injektion hin eine vermehrte Ausscheidung von Kreatin statt, aber dieselbe entspricht durchaus nicht der eingeführten Kreatinmenge, sondern stellt nur einen geringen Bruchteil derselben dar; berechnet man das Plus der ausgeschiedenen Kreatinmenge selbst zu 0,05 $\mathrm{g}$ - was sicherlich

Tabelle V.

Foxterrier $10 \mathrm{~kg}$; vor Beginn des Versuches 2 Hungertage.

\begin{tabular}{|c|c|c|c|c|c|c|}
\hline V.T. & $\begin{array}{c}\text { Urin- } \\
\text { menge } \\
\text { von } 6-12 \\
\text { und } \\
12-6 \text { Uhr } \\
\text { ccm }\end{array}$ & $\mathrm{N}$ & Kreatinin & Kreatin & $\begin{array}{l}\text { Gesamt- } \\
\text { wert für } \\
\text { Kreatinin } \\
\text { und } \\
\text { Krealin }\end{array}$ & Bemerkungen \\
\hline 1. & $\begin{array}{c}5,5 \\
\left(122^{-3}\right)\end{array}$ & 0,05769 & 0,04485 & - & 0,04485 & Hunger \\
\hline \multirow[t]{2}{*}{2.} & $\begin{array}{l}52 \\
22\end{array}$ & $\begin{array}{l}1,28150 \\
0,48550\end{array}$ & $\begin{array}{l}0,10852 \\
0,06854\end{array}$ & $\overline{0,00570}$ & $\begin{array}{l}0,10852 \\
0,07424\end{array}$ & \multirow[t]{2}{*}{ Hunger ${ }^{1}$ ) } \\
\hline & 74 & 1,76700 & 0,17706 & 0,00570 & 0,18276 & \\
\hline 3. & 17 & 0,59670 & 0,02152 & 0,00842 & 0,02994 & Hunger \\
\hline \multirow[t]{2}{*}{4.} & $\begin{array}{l}50 \\
23\end{array}$ & $\begin{array}{l}1,63400 \\
0,13870\end{array}$ & $\begin{array}{l}0,02746 \\
0,01982\end{array}$ & \multirow[t]{2}{*}{-} & $\begin{array}{l}0,02746 \\
0,01982 \\
\end{array}$ & \multirow{2}{*}{$\begin{array}{l}\text { Milch und } \\
\text { Brot }\end{array}$} \\
\hline & 73 & 1,77270 & 0,04728 & & 0,04728 & \\
\hline
\end{tabular}

1) $0,5 \mathrm{~g} \mathrm{Kreatin} \mathrm{in} 50 \mathrm{ccm}$ physiol. Kochsalzlösung intravenös. 
$\mathrm{zu}$ viel ist -, so bedeutet das ein Zehntel der eingeführten Menge. In Tabelle $\mathrm{V}$ erhält dasselbe Tier $0,5 \mathrm{~g}$ Kreatin in $50 \mathrm{ccm}$ physiologischer Kochsalzlösung intravenös. Eine Erläuterung der Werte der Kreatinrubrik ist überflüssig. Es fand sicher keine Vermehrung der ausgeschiedenen Kreatinmenge statt. Vor dem Beginn des Versuchs hatte das Tier 2 Tage lang gehungert. In Tabelle VI findet sich nach ebenfalls intravenöser Zufuhr von $0,5 \mathrm{~g}$ Kreatin wiederum eine deutliche Vermehrung des ausgeschiedenen Kreatins; es waren dem Versuche keine Hungertage vorausgegangen. Tabelle VII gibt die Werte bei einem hungernden Tier nach subkutaner Injektion von $0,3 \mathrm{~g}$ Kreatin wieder; im Gegensatz zu den Befunden der Tabelle $\mathrm{V}$ fand hier eine zweifellose Kreatinvermehrung statt; dabei muß jedoch hervorgehoben werden, daß es sich um ein ganz junges, etwa $3 / 4$ Jahre altes Tier handelte. In Tabelle VIII schließlich findet sich regelmäßig bei einem gut genährten Tiere

\section{Tabelle VI.}

Foxterrier $5 \mathrm{~kg}$. Fütterung mit Brot und Milch.

\begin{tabular}{|c|c|c|c|c|c|c|}
\hline V. T. & $\begin{array}{c}\text { Urin- } \\
\text { menge } \\
\text { von } \\
6-12 \\
12-6 \\
\text { Uhr } \\
\text { ccm }\end{array}$ & $\mathrm{N}$ & $\begin{array}{l}\text { Krea- } \\
\text { tinin }\end{array}$ & Kreatin & $\begin{array}{l}\text { Gesamt- } \\
\text { wert für } \\
\text { Krea- } \\
\text { tinin } \\
\text { und } \\
\text { Kreatin }\end{array}$ & Bemerkungen \\
\hline \multirow[t]{3}{*}{1.} & 180 & 1,22114 & 0,10055 & 一 & 0,10055 & \multirow{3}{*}{$\begin{array}{l}\text { Injektion von } 0,5 \mathrm{~g} \text { Kreatin } \\
\text { in die V. jugularis um } \\
1 \text { Uhr. Milch und Brot, } \\
\text { dann Hungertag. }\end{array}$} \\
\hline & $\mathbf{5 3}$ & 0.20166 & 0.07276 & 0..88334 & $\Omega, 1561 \Omega$ & \\
\hline & 233 & 1,42280 & 0,17331 & 0,08334 & 0,25665 & \\
\hline \multirow[t]{3}{*}{2.} & 144 & 1,04371 & 0,08640 & 0,01418 & 0,10058 & \multirow{3}{*}{ Hunger, } \\
\hline & 220 & 0,04276 & 0,04686 & 0,00998 & 0,05684 & \\
\hline & 364 & 1,08647 & 0,13326 & 0,02416 & 0,15742 & \\
\hline \multirow[t]{3}{*}{3.} & 100 & 2,20989 & 0,10519 & 0,06012 & 0,16531 & \multirow{3}{*}{ Milch und Brot. } \\
\hline & 36 & 0,85804 & 0,03204 & 0,00036 & 0,03240 & \\
\hline & 136 & 3,06793 & 0,13723 & 0,06048 & 0,19771 & \\
\hline
\end{tabular}

Hoppe-Seyler's Zeitschrift f. physiol. Chemie. LVII. 
Tabelle VII.

Bastardhündin $6750 \mathrm{~g}$. Seit 2 Tagen Hunger.

\begin{tabular}{|c|c|c|c|c|c|c|}
\hline V.T. & $\begin{array}{c}\text { Urin- } \\
\text { menge } \\
\text { von } \\
6-12 ; \\
12-6 \\
\text { Uhr } \\
\text { ccm }\end{array}$ & $\dot{N}$ & $\begin{array}{l}\text { Krea- } \\
\text { tinin }\end{array}$ & Kreatin & $\begin{array}{c}\text { Gesamt- } \\
\text { wert für } \\
\text { Krea- } \\
\text { tinin } \\
\text { und } \\
\text { Kreatin }\end{array}$ & Bemerkungen \\
\hline \multirow[t]{3}{*}{1.} & 39 & 0,92320 & 0,09212 & 0,03951 & 0,13163 & \multirow{3}{*}{ Hunger. } \\
\hline & 9 & 0,29553 & $\mid 0,02701$ & 0,02457 & $0,051 \check{8} 8$ & \\
\hline & 48 & 1,21873 & 0,11913 & 0,06408 & 0,18321 & \\
\hline \multirow[t]{3}{*}{2.} & 61 & 1,13830 & 0,10084 & 0,11399 & 0,21483 & \multirow{3}{*}{$\begin{array}{c}\text { Injektion von } 0,3 \mathrm{~g} \text { Kreatin } \\
\text { subkutan. Hunger. }\end{array}$} \\
\hline & 0 & - & - & - & - & \\
\hline & 61 & & & & & \\
\hline \multirow[t]{3}{*}{3.} & 43 & 1,15000 & $0,08: 331$ & 0,06213 & 0,15144 & \multirow{3}{*}{ Hunger. } \\
\hline & 22 & 0,64246 & 0,04950 & 0,03 ว̄55 & 0,08505 & \\
\hline & 65 & 1,79246 & 0,13881 & 0,09768 & 0,23649 & \\
\hline
\end{tabular}

nach Kreatinzufuhr auch Vermehrung der Kreatinausfuhr, allerdings in wechselnder Stärke. Bei der ersten, subkutanen Injektion wurde am meisten Kreatin ausgeschieden; bei der zweiten, ebenfalls subkutanen Injektion wurden $2 \mathrm{cg}$ Morphin. mur. mitinjiciert, ebenso bei der dritten, intravenösen Injektion. $\mathrm{Ob}$ diese verminderte Kreatinausscheidung auf die Morphingabe bei den Injektionen zurückzuführen ist, muß dahingestellt bleiben, bis weitere Untersuchungen hierüber vorliegen. Der Unterschied ist jedoch ein so auffälliger, daß man auf die Annahme eines ursächlichen Zusammenhangs geradezu hingedrängt wird.

Aus allen diesen Versuchen geht nun zunächst mit Sicherheit hervor, daß durch intravenöse oder subkutane Injektion zugeführtes Kreatin nur zum allergeringsten Teile wieder im Harn erscheint; beim länger hungernden Tiere scheint die Ausscheidung des eingeführten Kreatins noch viel geringer zu sein als bei Tieren, die bis zum Beginn der Versuche regelmäßig gefüttert wurden oder während derselben weitergefültert wurden. 
Beiträge zum Kreatininstoffwechsel.

Tabelle VIII.

Spitzhündin $7 \mathrm{~kg}$; seit 10 Tagen nur Milch und Brot.

\begin{tabular}{|c|c|c|c|c|c|c|}
\hline V. T. & $\begin{array}{c}\text { Urin- } \\
\text { menge } \\
\text { von } \\
6-12 \\
12-6 \\
\text { Uhr } \\
\text { ccm }\end{array}$ & N. & Kreatinin & Kreatin & $\begin{array}{l}\text { Gesamt- } \\
\text { wert für } \\
\text { Kreatinin } \\
\text { und } \\
\text { Kreatin }\end{array}$ & Bemerkungen \\
\hline \multirow[t]{3}{*}{1.} & $15 \check{3}$ & 1,34085 & 0,09682 & 0,02004 & 0,11686 & \multirow{3}{*}{$\begin{array}{l}\text { Um } 1 \text { Uhr Injektion } \\
\text { von } 0,5 \mathrm{~g} \text { Kreatin } \\
\text { subkutan in } 00 \mathrm{ccm} \\
\text { Kochsalzlösung. }\end{array}$} \\
\hline & 37 & 0,56111 & 0,07309 & 0,08465 & 0,15774 & \\
\hline & 190 & 1,90196 & 0,16991 & 0,10469 & 0,27460 & \\
\hline \multirow[t]{3}{*}{2.} & 275 & 1,94132 & 0,18562 & 0,01326 & 0,19888 & \multirow{3}{*}{ Milch und Brot. } \\
\hline & 235 & 0.6928 & 0,05734 & 0.00611 & $0,06: 345$ & \\
\hline & 510 & 2,63 任0 & 0,24296 & 0,019:37 & 0,26233 & \\
\hline \multirow[t]{3}{*}{3.} & 365 & 1,49148 & 0,16609 & 0,00989 & 0,17598 & \multirow{3}{*}{ Milch und Brot. } \\
\hline & 67 & 0,63778 & 0,05899 & 000446 & 0.06345 & \\
\hline & 432 & 2,12926 & 0,22508 & 0,01435 & 0,23943 & \\
\hline 4. & $\begin{array}{c}100 \\
6--12 \\
\text { Uhr }\end{array}$ & 1,04348 & 0,07365 & 0,00423 & 0,07778 & \\
\hline \multirow[t]{3}{*}{5.} & 190 & 1,92600 & 0,19239 & $一$ & 0,19239 & \multirow{3}{*}{$\begin{array}{l}\text { Um } 1 \text { Uhr } 0,5 \mathrm{~g} \text { Krea- } \\
\text { tin und } 0,4 \mathrm{~g} \text { Mor- } \\
\text { phin subkutan. }\end{array}$} \\
\hline & 20 & 0,46894 & 0,05684 & 0.04361 & 0.10045 & \\
\hline & 210 & 2,39494 & 0,24923 & 0,04361 & 0.29284 & \\
\hline \multirow[t]{3}{*}{6.} & 85 & 1,$092 ; 0 \mid$ & 0,13240 & 一 & 0,13240 & \multirow{3}{*}{ Milch und Brot. } \\
\hline & 28 & 0,46829 & 0,06131 & 0,00349 & 0.06480 & \\
\hline & 113 & 1,56099 & 0,19371 & $000: 349$ & 0.19720 & \\
\hline \multirow[t]{3}{*}{7.} & 60 & 0,85282 & 0,07146 & 0,00693 & 0,07839 & \multirow{3}{*}{ Milch und Brot. } \\
\hline & 28 & $0,5703 i$ & 0.06300 & 一 & 0,06300 & \\
\hline & 88 & 1.42317 & 0,13446 & 0,00693 & 0,14139 & \\
\hline 8. & $\left|\begin{array}{c}55 \\
6-12 \\
\text { Uhr }\end{array}\right|$ & 0,89730 & 0,06552 & 0,00409 & 0,06961 & Milch und Brot. \\
\hline \multirow[t]{3}{*}{9.} & 118 & 1,27403 & 0,16479 & 0,03433 & 0,19912 & \multirow{3}{*}{$\begin{array}{l}\text { Um } 4 \text { Uhr 0,5̃ Krea- } \\
\text { tin intravenös in } \\
\text { Morph.- Äthernar- } \\
\text { kose am 8. Tage. }\end{array}$} \\
\hline & 23 & 0,40459 & 0,06010 & 0.00098 & 0,06108 & \\
\hline & 141 & 1,67862 & 0,22489 & 0,03531 & 0,25020 & \\
\hline 10. & $\begin{array}{c}55 \\
6-12 \\
\text { Uhr }\end{array}$ & 1,06100 & 0,11137 & 一 & 0,11137 & Milch und Brot. \\
\hline 11. & $\left|\begin{array}{c}\frac{44}{12}-12 \\
\text { Uhr }\end{array}\right|$ & 0,75553 & 0,09504 & 一 & 0,09504 & Milch und Brot. \\
\hline
\end{tabular}


Aber in keinem Falle entsprach die Kreatinausscheidung auch nur annähernd dem eingeführten Kreatinquantum; die Menge des vermehrt ausgeschiedenen Kreatins betrug stets nur einen verschwindenden Teil des injizierten Kreatins ganz im Gegensatz zu den Fütterungsversuchen mit Fleischextrakt. Wo blieb die nicht ausgeschiedene Kreatinmenge? Man kann natürlich mit Folin annehmen, daß sie im Körper retiniert ward, dann erhebt sich die weitere Frage nach dem Ort der Anlagerung. Nach Mellanby findet im Muskel keine Anlagerung statt. Das Kreatin müßte also in anderen Organen zurückgehalten worden sein. $O b$ das tatsächlich der Fall ist und ob dies überhaupt möglich ist, darüber geben die Versuche keinen Aufschluß. Aber auch eine andere Möglichkeit muß ernstlich in Erwägung gezogen werden: die Zerstörung des Kreatins. Wie Gottlieb und Stangassinger gezeigt haben, findet bei der Autolyse aller Organe eine Zerstörung zugesetzten Kreatins statt durch ein Ferment, die Kreatase, und es ist sehr wohl denkbar, daß durch die Wirkung desselben die geringe Ausscheidung des eingeführten Kreatins zustande kommt.

Allerdings beobachtet man keine vermehrte Stickstoffausfuhr. Es ist aber die Möglichkeit ohne weiteres zuzugeben, daß numal heim hungernden Tiare der Stickssfoff, dor ous dom Zerfall des Kreatins entsteht, assimiliert wird, und damit wäre zugleich auch der Widerspruch erklärt, daß Fleischextrakt die Kreatinausfuhr vermehrt, Kreatingaben aber nicht. Der Fleischextrakt enthält genügend Stickstoff, um den Bedarf des Organismus zu decken, sodaß eine Aufteilung des Kreatins unnötig wird, mit anderen Worten: Eingeführtes Kreatin wird erst ausgeschieden, wenn für den Organismus kein Bedarf an Stickstoff vorliegt. Auf anderem Wege ist bereits Folin zu dieser Schlußfolgerung gelangt, ohne jedoch den Vorgang der Kreatasewirkung, wie sie allerdings erst durch die Versuche von Gottlieb und Stangassinger erkannt wurden, in Erwägung zu ziehen.

Eine andere Möglichkeit des Zustandekommens der gleichmäßigen Kreatinausfuhr trotz Einverleibung größerer Kreatinmengen, die Überführung von Kreatin zu Kreatinin blieb bis-- 
her gänzlich unberücksichtigt, da sich nach unseren Versuchen nicht der geringste Anhalt dafür bietet, daß parenteral zugeführtes Kreatin durch den Hundeorganismus in Kreatinin verwandelt wird. Die Menge des ausgeschiedenen Kreatinins zeigte bei allen unseren Versuchen so gut wie keine Schwankung.

VI. Die Kreatinin- und Kreatinausscheidung bei der Leberschädigung.

Trotz sehr umfangreicher Studien ist die Funktion der Leber bei dem Kreatinin- und Kreatinstoffwechsel noch nicht völlig geklärt. Bisher ist ruur bekannt, daß die Leber im Kreatinin- und Kreatinhaushalt eine bedeutende Rolle spielt. Es geht dies in allererster Linie auch aus den Untersuchungen Mellanbys über die Funktion der Leber für den Kreatinstoffwechsel während des Wachstums hervor. Aber ganz klar gestellt sind die Verhältnisse wie erwähnt noch nicht. Es liegt dies, wohl daran, daß in der Leber nicht nur ein für den Kreatinin- und Kreatinstoffwechsel bedeutsamer Prozeß vor sich geht, sondern mehrere. Wie Gottlieb und Stangassinger gezeigt haben, hat der Leberpreßsaft die Fähigkeit, sowohl aus zugesetztem Kreatin Kreatinin zu bilden, als auch umgekehrt und außerdem sowohl Kreatin wie Kreatinin zu zerstören.

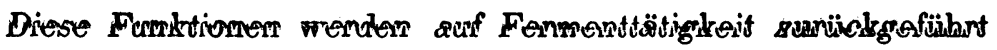
und sind durch Autolyseversuche festgestellt worden. Ob nun intra vitam alle diese Funktionen gleichmäßig zur Geltung kommen, oder unter welchen Bedingungen die eine etwas stärker zum Ausdruck kommt als die andere, ist bisher nur durch die von Gottlieb und Stangassinger angestellten Durchleitungsversuche geprüft worden. Trotzdem erschwert die Tatsache, daß nach den Versuchen von Gottlieb und Stangassinger dem Leberextrakte so völlig entgegengesetzte Fermentwirkungen zu eigen sind, die Deutung aller Versuchsresultate nach Leberschädigung ganz ungeheuer. Das fiel auch Mellanby, der allerdings die Durchleitungsversuche noch nicht kannte, bei seinen Beobachtungen an Leberkranken auf und ist der Grund, warum er mehrere Möglichkeiten bei der Deutung seiner Befunde in Erwägung zieht. Dazu kommt aber noch eine weitere Schwierigkeit bei der Bewertung von Untersuchungsergebnissen, 
die man nach Leberschädigung erhält. Es ist geradezu unmöglich, Leberschädigungen hervorzurufen, welche nur die Funktion der Leber treffen, ohne das Leberparenchym anzugreifen. Meistens wird sogar außer der Leber auch der übrige Organismus nicht verschont bleiben. Eine dritte Schwierigkeit besteht darin, genau den Zeitpunkt anzugeben, wann wirkiich eine Schädigung der Leber stattgefunden hat. Am meisten Chancen bot hierfür die Urobilinreaktion; für die gewünschten Aufschlüsse war aber auch diese leider nicht ausreichend. So stehen den Versuchen, die Kreatinin- und Kreatinausscheidung nach Leberschädigung zu studieren, eine Reihe von sehr beträchtlichen Schwierigkeiten im Wege. Da aber keine andere Möglichkeit übrig blieb, um intra vitam den Anteil der Leber an dem Kreatinin- und Krealinstoffwechsel zu untersuchen, falls man nicht einen Weg einschlug, der Vergleiche der beim Tiere erhaltenen Resultate mit den im Menschen zu gewinnenden von vorneherein ausschloß - wie z. B. Ausschaltung der ganzen Leber -, so wurde der Versuch gemacht, die Leber durch verschieden stark wirkende Agenzien zu schädigen und dadurch ihren Einfluß auf den Kreatinin- und Kreatinstoffwechsel $z u$ ermitteln.

Bekanntlich entsteht nach der Finverleihung von Amylalkohol in den Organismus ein typisches Krankheitsbild, das sich durch leichte Narkose und Brechreiz namentlich auszeichnet und am meisten Ähnlichkeit mit einem schweren Rausche hat. Im Anschluß an dieses akute Vergiftungsstadium beobachtet man nach mehrmaligen, genügend großen Dosen von Amylalkohol Auftreten von Urobilin im Urin, was als sicheres Zeichen einer bestehenden Leberschädigung angesehen werden darf. In Tabelle IX sind die Werte wiedergegeben, die bei einem $10 \mathrm{~kg}$ schweren Tiere nach Amylalkoholgaben erhalten wurden. Das Tier war bereits, als die Vergiftungsversuche begannen, mehrere Tage vorher ausschließlich mit Brot und Milch gefüttert worden. Der Amylalkohol, erst $4 \mathrm{ccm}$, darn $10 \mathrm{ccm}$, wurde stets mit der Schlundsonde unter Beifügung von 20 bezw. $30 \mathrm{ccm}$ Äthylalkohol eingegossen. Gewöhnlich war der "Rauschzustand» ein so schwerer, daß das Tier nur wenig 
Beiträge zum Kreatininstoffwechsel.

Tabelle IX.

Hündin $10 \mathrm{~kg}$; seit einer Woche mit Brot und Milch ernährt.

\begin{tabular}{|c|c|c|c|c|c|c|}
\hline V. T. & $\begin{array}{c}\text { Urin- } \\
\text { menge } \\
\text { von } \\
6-12 \\
12-6 \\
\text { Uhr } \\
\text { ccm }\end{array}$ & $\mathrm{N}$ & $\begin{array}{l}\text { Krea- } \\
\text { tinin }\end{array}$ & Kreatin & $\begin{array}{c}\text { Gesamt. } \\
\text { wert für } \\
\text { Krea- } \\
\text { tinin } \\
\text { und } \\
\text { Kreatin }\end{array}$ & Bemerkungen \\
\hline \multirow[t]{3}{*}{1.} & 140 & 0,91128 & 0,09009 & 0,02331 & 0,11340 & \multirow{3}{*}{$\begin{array}{c}\text { Brot und Milch und } 5 \mathrm{~g} \\
\text { Liebigs Fleischextrakt. }\end{array}$} \\
\hline & 39 & 0,45337 & 0,07421 & - & 0,07521 & \\
\hline & 179 & 1,36465 & 0,16530 & 0,02331 & $0,18761 \mid$ & \\
\hline 2. & $\left|\begin{array}{c}178 \\
6-12\end{array}\right|$ & 1,66042 & 0,09115 & 0,05297 & 0,14412 & \\
\hline \multirow[t]{3}{*}{3.} & 50 & 0,75744 & 0,09642 & 0,01158 & 0,10800 & \multirow{3}{*}{$\begin{array}{l}\text { Um } 1 \text { Uhr } 4 \mathrm{ccm} \text { Amyl } \\
\text { alkohol mit } 20 \mathrm{ccm} \text { Äthyl } \\
\text { alkohol. }\end{array}$} \\
\hline & 70 & 1.10113 & 0,14920 & 0.02799 & 0,17719 & \\
\hline & 120 & 1,85857 & 0,24562 & 0,03957 & 0,28519 & \\
\hline \multirow[t]{3}{*}{4.} & 85 & 1,2049 & 0,07325 & 0,07642 & 0,14967 & \multirow{3}{*}{$\begin{array}{l}\text { Brot und Milch und } 5 g \\
\text { Liebigs Fleischextrakt }\end{array}$} \\
\hline & 40 & - & 0,07535 & 0,01465 & 0,09000 & \\
\hline & 125 & 1,2049 & 0,14860 & 0,09107 & 0,23967 & \\
\hline \multirow[t]{3}{*}{5.} & 275 & $2,50500 \mid$ & 0,27165 & $0,029: 36$ & 0,30101 & \multirow{3}{*}{$\begin{array}{l}\text { Um } 1 \text { Uhr } 4 \text { ccm Amyl } \\
\text { alkohol und } 10 \mathrm{ccm} \text { Äthyl } \\
\text { alkohol. Nahrung wio am } \\
\text { 4. Tage. }\end{array}$} \\
\hline & 90 & 0,33358 & 0,04095 & 0.02782 & 0,06877 & \\
\hline & 365 & 2,85858 & $0,5 \mathrm{r} 26 \mathrm{v}$ & 0,05718 & 0,36978 & \\
\hline \multirow[t]{3}{*}{6.} & 140 & 1,73604 & 0,20250 & 0,01558 & 0,21808 & \multirow{3}{*}{ Nahrung wie am 4. Tage. } \\
\hline & 50 & 1,06424 & $0,1 \cap 125$ & 0,00533 & 0.10658 & \\
\hline & 190 & 2,80028 & 0,30375 & 0,02091 & 0,32466 & \\
\hline \multirow[t]{3}{*}{7.} & 45 & 0,50529 & 0,08476 & 0,00636 & 0,09112 & \multirow{3}{*}{ Nahrung wie am 4. Tage. } \\
\hline & 70 & 0,71038 & 0,09776 & 0,00724 & $0,105 \cap 0$ & \\
\hline & 115 & 1,21567 & 0,18252 & 0,01360 & 0,19612 & \\
\hline 8. & $\left|\begin{array}{c}120 \\
12-12\end{array}\right|$ & 1,79773 & 0,24300 & 0,02700 & 0,27000 & do. \\
\hline 9. & $\left|\begin{array}{c}82 \\
6-12\end{array}\right|$ & 0,82962 & 0,16605 & 0,00874 & 0,17479 & do. \\
\hline \multirow[t]{3}{*}{10.} & 300 & $2,432: 33$ & 0,15379 & 0,14996 & 0,30375 & \multirow{3}{*}{ do. } \\
\hline & 45 & 0,38185 & 0,03779 & 0,00894 & 0,04673 & \\
\hline & 345 & 2,81418 & 0,19158 & 0,15890 & 0,35048 & \\
\hline
\end{tabular}


Fortsetzung von Tabelle IX.

\begin{tabular}{|c|c|c|c|c|c|c|}
\hline V.T. & $\begin{array}{c}\text { Urin- } \\
\text { menge } \\
\text { von } \\
6-12 \\
12-6 \\
\text { Uhr } \\
\text { ccm }\end{array}$ & $\mathrm{N}$ & $\begin{array}{l}\text { Krea- } \\
\text { tinin }\end{array}$ & Kreatin & $\begin{array}{l}\text { Gesamt- } \\
\text { wert für } \\
\text { Krea- } \\
\text { tinin } \\
\text { und } \\
\text { Kreatin }\end{array}$ & Bemerkungen \\
\hline \multirow[t]{3}{*}{11.} & 500 & 4,27660 & 0,23012 & 0,03287 & 0,26299 & \multirow{3}{*}{$\begin{array}{l}\text { Kein Fleischextrakt mehr. } \\
\text { Um } 1 \text { Uhr } 10 \mathrm{ccm} \text { Amyl-- } \\
\text { alkohol und } 30 \mathrm{ccm} \text { Äthyl-- } \\
\text { alkohol. }\end{array}$} \\
\hline & 108 & 0,40759 & 0,03390 & 0.00660 & 0,04050 & \\
\hline & 608 & 4,68419 & 0,26402 & 0,03947 & 0,30349 & \\
\hline \multirow[t]{3}{*}{12.} & 176 & 2,40383 & 0,19285 & 0,01081 & 0,20366 & \multirow{3}{*}{ Milch und Brot. } \\
\hline & 58 & 1,29185 & 0,06909 & 0,00432 & 0,07341 & \\
\hline & 234 & $\mid 3,69568$ & $|0,26194|$ & 0,01513 & 0,27707 & \\
\hline \multirow[t]{3}{*}{13.} & 365 & 3,04814 & 0,23464 & 0,04428 & 0,27892 & \multirow{3}{*}{ Milch und Brot. } \\
\hline & 230 & 0,89838 & 0,05146 & 0,02366 & 0,07512 & \\
\hline & 595 & $|3,94652|$ & 0,28610 & 0,06794 & 0,35404 & \\
\hline \multirow[t]{3}{*}{14.} & 218 & 1,12637 & 0,22073 & 0,01790 & 0,23863 & \multirow{3}{*}{$\begin{array}{l}\text { Um } 1 \text { Uhr } 10 \mathrm{ccm} \text { Amyl-. } \\
\text { alkohol und } 30 \mathrm{ccm} \text { Äthyl-. } \\
\text { alkohol. }\end{array}$} \\
\hline & $7 \check{5}$ & 0,69667 & 0,05346 & 0,00610 & 0,05956 & \\
\hline & 293 & 1,82304 & 0,27419 & 0,02400 & 0,29819 & \\
\hline \multirow[t]{3}{*}{ 15. } & 270 & 2,55653 & 0,22116 & $0,03: 314$ & 0,25430 & \multirow{3}{*}{ Spur Urobilin. } \\
\hline & 43 & 0,28085 & 0,02097 & 0,00194 & 0,02291 & \\
\hline & 313 & 2,83738 & 0,24213 & 0,03508 & 0,27721 & \\
\hline \multirow[t]{3}{*}{16.} & 305 & 2,85031 & 0,19301 & 0,03475 & 0,22776 & \multirow{3}{*}{ Um 6 Uhr $0,05 \mathrm{~g}$ Phosphor. } \\
\hline & 42 & 0,89043 & 0,05854 & 0,01223 & 0,07087 & \\
\hline & 347 & 3,75074 & 0,25165 & 0,04698 & 0,29863 & \\
\hline \multirow[t]{3}{*}{17.} & 429 & 2,50088 & 0,16088 & 0,04096 & 0,20184 & \multirow{3}{*}{ Reichlich Urobilin. } \\
\hline & 138 & 1,09510 & 0,05762 & - & 0,05762 & \\
\hline & 567 & 3,59598 & 0,21850 & $0,04096 \mid$ & 0,25946 & \\
\hline \multirow[t]{3}{*}{18.} & 60 & $0,188: 36$ & 0,01065 & 0,00019 & 0,01084 & \multirow{3}{*}{ Um 6 Uhr 0,075 g Phosphor. } \\
\hline & 110 & 0,51767 & 0,02357 & 0,00174 & 0,02531 & \\
\hline & 170 & 0,70603 & 0,03422 & 0,00193 & 0,03615 & \\
\hline \multirow[t]{3}{*}{19.} & 200 & 0,79748 & 0,04793 & 0,00028 & 0,04821 & \multirow{3}{*}{ Um 6 Uhr $0,1 \mathrm{~g}$ Phosphor. } \\
\hline & 32 & 0,27082 & - & - & $\underline{0,01580}$ & \\
\hline & 232 & 1,06830 & 0,04793 & 0,00028 & 0,06401 & \\
\hline 20. & 305 & 2,54618 & 0,09429 & 0,00779 & 0,10208 & Exitus. \\
\hline
\end{tabular}


erbrach, völlig ataktisch war und auf äußere Reize kaum reagierte. Erst schmerzhafte Manipulationen - kneifen mit der Zange - zeigten, daß die Narkose keine vollständige war. Im Beginn der Versuche wurden, um zugleich eine Belastung des Organismus herbeizuführen, außer der Milch- und Brotdiät, noch täglich $5 \mathrm{~g}$ Fleischextrakt verfüttert. Am Mittag des 3. Versuchstages wurden zum ersten Male $4 \mathrm{ccm}$ Amylalkohol eingegossen; daraufhin ging ohne wesentliche Steigerung des $\mathrm{N}$ die Kreatininausscheidung deutlich in die Höhe; die Kreatinausscheidung blieb gleich. Am nächsten Tage sank die Kreatininmenge etwas; dafür stieg die Kreatinmenge deutlich an, sodaß der Gesamtwert kaum einen Rückgang zeigte. Am nächsten, dem 5. Versuchstage, wurden $10 \mathrm{ccm}$ Amylalkohol eingegossen; mit steigendem Stickstoffwert ging der Kreatininwert in die Höhe, während die Kreatinausscheidung gleich blieb. Am nächsten Tage bot sich dasselbe Bild dar; vom 7. bis 11. Tage sank dann bei wechselndem $\mathrm{N}$ der Kreatin- und Kreatininwert etwas. Vom 11. bis 16. Versuchstage waren bei hohen Diuresezahlen und hohen Stickstoffwerten die Kreatininund Kreatinwerte gleich, obwohl zweimal, am 11. und 14. Tage, je $10 \mathrm{ccm}$ Amylalkohol verabreicht wurden. Am 16. Tage abends wurden 0,05 \& Phosphor eingegossen: es geschah dies in der Weise, daß von einer ${ }^{1} / 2 \%$ igen Phosphorolivenöllösung $10 \mathrm{ccm}$ mit einigen Tropfen Sodalösung und Wasser zu einer feinen, milchigen Emulsion verrührt wurden und mit der Schlundsonde eingegeben wurden. Am nächsten, dem 17. Tage, blieb die Kreatininausscheidung noch gleich, ging dann aber am 18. Tage deutlich und erheblich zurück. Am gleichen Tage wurden aberabermals $0,075 \mathrm{~g}$ Phosphor gegeben; daraufhin trotz hoher Stickstoffausscheidung geringe Kreatinin- und Kreatinausfuhr. Am Abend des 19. Tages nochmals $0,10 \mathrm{~g}$ Phosphor; infolgedessen trotz sehr hoher Stickstoffzahl im Urine geringe Kreatinin- und Kreatinmengen und Tod des Tieres. Es fand also eine wesentliche Änderung der Kreatinin- und Kreatinausfuhr durch Amylalkohol- und Äthylalkoholgaben trotz sicherer Leberschädigung, die durch das Auftreten der Uröbilinreaktion festgestellt war, nicht statt; erst die Phosphorvergiftung führte 
eine deutliche Verminderung der Kreatinin- und Kreatinausscheidung herbei. Dieselbe verlief allerdings ziemlich rasch; es kam nicht zu ausgedehntem Eiweißzerfall, wie dies aus den Stickstoffzahlen deutlich hervorgeht. In Tabelle X und XI sind die Ergebnisse von zwei weiteren Phosphorvergiftungsversuchen niedergelegt. Die beiden verwendeten Tiere waren sich an Körpergewicht ziemlich gleich. Das in Tabelle XI registrierte Tier erhielt jedoch etwas mehr Phosphor als das in Tabelle X, sodaß hier die Vergiftung einen etwas foudroyanteren Verlauf nahm, obwohl beide Tiere gleich lange am Leben blieben. Bei Betrachtung der Tabelle $\mathrm{X}$ fällt zunächst auf, daß mit steigendem Stickstoff auch die Kreatininwerte steigende Tendenz vom 1. bis 7. Tage zeigen. Die Kreatinwerte bleiben sich dagegen ziemlich gleich; erst vom 7 . Tage an, wo die Kreatininwerte im Rückgange begriffen sind, nehmen dieselben zu. Der zweite Tageswert des 8. Tages sowie derjenige des 9. Tages übersteigen den Kreatininwert, ein sicheres, pathologisches Zeichen. Mit dem Rückgange der Kreatininwerte findet auch eine Verminderung der Gesamtausscheidung von Kreatinin und Kreatin trotz Anstieg des letzteren statt. Wir finden also mit steigendem Stickstoff einen Anstieg der Kreatininausscheidungsoröße bei gleichbleibendem Kreatin, mit abnehmendem Stickstoff und abnehmendem Kreatinin Zunahme der Kreatinausfuhr. Noch deutlicher kommt dieses Verhältnis in Tabelle XI zum Ausdruck. Hier beobachteten wir unter dem Einfluß der Phosphorwirkung eine kolossale Zunahme des Stickstoffes; damit Hand in Hand Zunahme der Kreatininausscheidung. Am 7. Tage erreichen beide ihre Höhe; zugleich wird die Kreatinmenge der Kreatininmenge gleich. Vom 8. Tage nimmt die ausgeschiedene Stickstoffmenge ab, mit ihr die Kreatininmenge, und die Kreatinausfuhr steigt bis fast aufs Doppelte ihrer früheren Größe. - Unter dem Einfluß der Phosphorvergiftung entwickeln sich im Organismus hauptsächlich zwei Vorgänge: Enormer Eiweißverfall, namentlich auch des Blutes, verbunden mit verminderter Alkalescenz und Vermehrung von Gallenfarbstoffen und Fettdegeneration und Fettinfiltration der Leber, später auch Schädigung der Nieren. Als Symptom der 
Beiträge zum Kreatininstoffwechsel.

Tabelle $\mathrm{X}$.

Spitzhündin $7 \mathrm{~kg}$. Seit 2 Tagen Hunger.

\begin{tabular}{|c|c|c|c|c|c|c|}
\hline V. T. & $\begin{array}{c}\text { Urin- } \\
\text { menge } \\
\text { von } \\
6-12 ; \\
12-6 \\
\text { Uhr } \\
\mathrm{ccm}\end{array}$ & $\mathrm{N}$ & Kreatinin & Kreatin & $\begin{array}{c}\text { Gesaint- } \\
\text { wert für } \\
\text { Kreatinin } \\
\text { und } \\
\text { Kreatin }\end{array}$ & Bemerkungen \\
\hline 1. & 25 & 0,36110 & 0,06136 & - & 0,06428 & Hunger. \\
\hline 2. & 43 & 0,27000 & 0,10801 & - & 0,10801 & Hunger. \\
\hline 3. & 64 & 0,55065 & 0,0684:3 & 0,01601 & 0,084444 & Milch und Brot. \\
\hline \multirow[t]{2}{*}{4.} & $\begin{array}{r}220 \\
43 \\
\end{array}$ & $\begin{array}{l}2,50903 \\
0,39411 \\
\end{array}$ & $\begin{array}{l}0,15496 \\
0,04050 \\
\end{array}$ & $\begin{array}{c}0,03262 \\
- \\
\end{array}$ & $\begin{array}{l}0,18758 \\
0,04050 \\
\end{array}$ & \multirow{2}{*}{$\begin{array}{l}\text { 0,04 g Psosphor. } \\
\text { Milch und Brot. }\end{array}$} \\
\hline & 263 & 2,90314 & 0,19546 & 0,03262 & 0,22808 & \\
\hline \multirow[t]{3}{*}{5.} & 87 & 0,59070 & $0,10 \check{95}$ & 0,00970 & 0,11565 & \multirow{3}{*}{$\begin{array}{l}\text { Um } 1 \text { Uhr } 0,04 \mathrm{~g} \\
\text { Phosphor. }\end{array}$} \\
\hline & 75 & 0,45405 & 0,03948 & 0,00049 & 0,03997 & \\
\hline & 162 & 1,04475 & 0,14543 & 0,01019 & 0,15562 & \\
\hline \multirow[t]{3}{*}{6.} & 150 & 1,58920 & 0,18135 & 0,00849 & 0,18984 & \multirow{3}{*}{ Urin Spur Urobilin. } \\
\hline & 53 & 0,65633 & 0.053366 & 0,01343 & 0,06709 & \\
\hline & 203 & 2,24553 & 0,23501 & 0,02192 & 0,25693 & \\
\hline \multirow[t]{3}{*}{7.} & 227 & 3,03440 & 0,28289 & 0,07764 & 0,36053 & \multirow{3}{*}{$\begin{array}{r}\text { Um6 Uhram6.Tage } \\
\text { 0,04 g Phosphor. }\end{array}$} \\
\hline & 65 & 0,69722 & 0,04920 & 0,01650 & 0,06570 & \\
\hline & 292 & 3,73162 & 0,33209 & 0,09414 & 0,42623 & \\
\hline \multirow[t]{3}{*}{8.} & 114 & 1,15570 & 0,12825 & 0,01603 & 0,14428 & \multirow{3}{*}{$\begin{array}{l}\text { Um } 1 \text { Uhr } 0,06 \mathrm{~g} \\
\text { Phosphor. }\end{array}$} \\
\hline & 49 & 0,34400 & 0,04961 & 0,05453 & 0,10414 & \\
\hline & 163 & 1,49970 & 0,17786 & 0,07056 & 0,24842 & \\
\hline 9. & 68 & 0,34600 & 0,02118 & 0,03740 & 0,05858 & Exitus. \\
\hline
\end{tabular}

Vergiftung unserer Tiere beobachteten wir namentlich an den beiden letzten Versuchstieren (Tab. X und XI) kolossale $\mathrm{Zu}$ nahme des Harnstickstoffes. Als weitere Folge des Eiweißverfalles ist wohl die ebenfalls bei den beiden letzten Tieren augenfällig in Erscheinung getretene Zunahme der Kreatininausfuhr 
Tabelle XI.

Vorstehhündin $6600 \mathrm{~g}$. Seit 2 Tagen Hunger. Dann Milch und Brot.

\begin{tabular}{|c|c|c|c|c|c|c|}
\hline V.T. & $\begin{array}{c}\text { Urin- } \\
\text { menge } \\
\text { von } \\
6-12 ; \\
12-6 \\
\text { Uhr } \\
\text { ccm }\end{array}$ & $\mathrm{N}$ & Kreatinin & Kreatin & $\begin{array}{c}\text { Gesamt- } \\
\text { wert für } \\
\text { Kreatinin } \\
\text { und } \\
\text { Kreatin }\end{array}$ & Bemerkungen \\
\hline 1. & $\begin{array}{c}26 \\
12-6 \\
\mathrm{Uhr}\end{array}$ & 0,73810 & 0,09142 & 0,01942 & 0,11084 & \\
\hline 2. & $1: 3$ & 0,09242 & 0,29941 & 0,035994 & 0,33535 & \\
\hline \multirow[t]{3}{*}{3.} & 80 & 0,12793 & 0,19638 & 0,08536 & 0,28174 & \multirow{3}{*}{$\begin{array}{c}0,04 \mathrm{~g} \text { Phosphor } \\
\text { um } 1 \text { Uhr. }\end{array}$} \\
\hline & 85 & 0,14360 & 0,13500 & 0,04619 & 0,18119 & \\
\hline & $16 \check{5}$ & 0,27153 & 0,33138 & 0,13155 & 0,46293 & \\
\hline \multirow[t]{3}{*}{4.} & 212 & 3,2833 & 0,27257 & 0,09280 & 0,36537 & \\
\hline & 42 & 0,9720 & 0,07560 & 0,02160 & 0,09720 & \\
\hline & 254 & 4,2553 & 0,34817 & 0,11440 & 0,46257 & \\
\hline \multirow[t]{3}{*}{5.} & 578 & 4,2913 & 0,30012 & 0,09003 & 0,39015 & \multirow{3}{*}{$\begin{array}{c}0,05 \mathrm{~g} \text { Phosphor } \\
\text { um } 1 \text { Uhr. }\end{array}$} \\
\hline & 160 & 1,7172 & 0,11172 & 0,01299 & 0,12462 & \\
\hline & .39 & 8,0095 & $0, \pm 1101$ & $0,1020: 3$ & 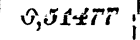 & \\
\hline \multirow[t]{3}{*}{6.} & 395 & 4,5500 & 0,24612 & 0,21096 & 0,45708 & \multirow{3}{*}{$\begin{array}{l}\text { Um } 6 \text { Uhr } 0,06 \mathrm{~g} \\
\text { Phosphor. }\end{array}$} \\
\hline & 86 & 1,9633 & 0,08293 & 0,03249 & 0,11542 & \\
\hline & 481 & 6,5133 & 0,32905 & 0,24345 & 0,57250 & \\
\hline \multirow[t]{3}{*}{7.} & 220 & 6,2150 & 0,32400 & 0,33600 & 0,66000 & \\
\hline & 80 & 1,8760 & 0,09818 & 0,09242 & 0,19060 & \\
\hline & 300 & 8,0910 & 0,42218 & 0,42842 & 0,85060 & \\
\hline \multirow[t]{3}{*}{8.} & 140 & 2,8347 & 0,16926 & 0,23574 & 0,40500 & \multirow{3}{*}{$\begin{array}{c}\text { Um } 1 \text { Uhr } 0,08 \mathrm{~g} \\
\text { Phosphor. }\end{array}$} \\
\hline & 67 & 1,3575 & 0,07434 & 0,11948 & 0,19382 & \\
\hline & 207 & 4,1922 & 0,24360 & 0,35522 & 0,59882 & \\
\hline \multirow[t]{3}{*}{9.} & 390 & 3,8000 & 0,26107 & 0,54893 & 0,81000 & \multirow{3}{*}{ Exitus. } \\
\hline & 18 & 0,2576 & 0,00219 & 0,07035 & 0,07254 & \\
\hline & 408 & 4,05576 & 0,26326 & 0,61928 & 0,88254 & \\
\hline
\end{tabular}


anzusehen, die mit der Stickstoffvermehrung Hand in Hand geht. Solange diese beiden Symptome beobachtet werden konnten, blieb die Kreatinmenge unverändert. Erst als sowohl der Stickstoff als die Kreatininausfuhr abnahm, trat Kreatinvermehrung auf. Diese Tatsache drängt geradezu zu der Annahme, daß die Leber, das bei der Phosphorvergiftung am meisten in Mitleidenschaft gezogene Organ, der Ort ist, wo die Aufspaltung des Eiweißes zu Kreatinin stattfindet; zum mindesten jedoch zu der Annahme, daß die Leber an der Kreatininbildung hervorragenden Anteil nimmt. $\mathrm{Zu}$ der gleichen Zeit, wo die Stickstoffmenge des Urines trotz fortgesetzter Vergiftung abnimmt, wo also der Organismus und wieder an erster Stelle die Leber nicht mehr imstande ist, das zerfallene Eiweiß völlig aufzuspalten, nimmt auch die Kreatininmenge ab und steigt die Kreatinmenge. Damit wird man zu der Annahme gezwungen, daß zwei Prozesse gestört sind: Einmal die Aufspaltung des zerfallenen Eiweißes zu Kreatinin, ferner die Beziehungen von Kreatinin zu Kreatin. Die vermehrte Ausscheidung von Kreatin wiederum läßt sich durch zwei Annahmen erklären: Entweder handelt es sich einfach dabei um Kreatinin, das durch die veränderte Reaktion des Harnes in Kreatin übergeführt und als

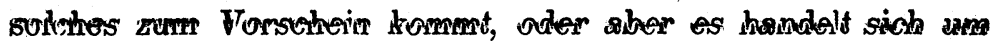
Kreatinmengen, die unter normalen Umständen angelagert oder zerstört werden und bei der Phosphorvergiftung in den Urin übergehen. Bekanntlich gehen fast alle Eiweißabbauprozesse in der Leber vor sich und es liegt deshalb nahe, auch die genannten Vorgänge in die Leber $\mathrm{zu}$ verlegen und nicht in die Nieren, obwohl man nach den Autolyseversuchen annehmen muß, daß die Nieren die gleichen Fermente enthalten wie die Leber, und obwohl im Verlaufe der Phosphorvergiftung auch die Nieren sicher geschädigt werden.

Eine Entscheidung über die Lokalisation der vermehrten Kreatininbildung bei Phosphorvergiftung ist nur dadurch zu treffen, daß man auch Nierenschädigungsversuche vornimmt, wie solche weiter unten folgen. Aus denselben geht jedoch, wie schon hier erwähnt sein mag, hervor, daß mit zunehmender Nierenschädigung keine Verminderung des Gesamtkreatinins 
und Kreatins stattfindet, daß also die Aufspaltung des Eiweißes und die Bildung von Kreatinin nicht in die Niere, sondern in die Leber zu verlegen ist, und daß dieselbe auf hört, wenn, wie in den beschriebenen Fällen, Insufficienz der Leber eintritt. Mit dieser Auffassung stimmen auch die Befunde überein, die Mellanby an seinen Kranken erhoben hat: daß Leberkranke im allgemeinen abnorm kleine Kreatininmengen ausscheiden, daß jedoch Leberkrebskranke große Kreatinmengen ausscheiden. Der erste Teil der von Mellanby gemachten Beobachtungen ist ohne weiteres erklärlich. Wenn die Leberfunktion insufficient wird, so wird weniger Kreatinin gebildet und ausgeschieden. Weniger klar liegen die Verhältnisse bei der vermehrten Kreatinausscheidung seiner Krebskranken. Nach Erwägung vieler Möglichkeiten kommt er zu der Annahme, daß bei der Kachexie der Kranken und dem Zugrundegehen von kreatinhaltiger Substanz (Muskelsubstanz) Kreatin frei wird, das ebenso wie verfüttertes als solches den Körper verläßt. $\mathrm{Ob}$ diese Deutung richtig ist, soll dahingestellt bleiben. Jedenfalls findet Mellanby ebenfalls bei Leberschädigungen Störungen in der Kreatininbildung als Folge der Insufficienz der Leber.

\section{Die Kreatinin- and Kreatinausscheidung nach Nierenschädigung.}

Wie aus der Besprechung der Resultate nach Phosphorvergiftung hervorgeht, waren die hierbei erhobenen Befunde insofern keine unbedingt eindeutige, als unter dem Einfluß der Phosphorwirkung bekanntlich auch die Nieren leiden. Es erschien daher zweckmäßig, auch Nierenschädigungsversuche vorzunehmen und den Einfluß derselben auf die Kreatinin- und Kreatinausscheidungen festzustellen. Zu diesem Zwecke wurden zwei Tiere mit Kaliumchromat behandelt. Nach den Angaben von S. Weber ${ }^{1}$ ) erhält man auf geringe Gaben von Kaliumchromat bei Hunden Albuminurie und Cylindrurie und es erschien deshalb das Kaliumchromat zu sicherer Nierenschädigung sehr geeignet. Es wurden wechselnde Mengen davon subkutan

1) S. Weber, Exper. Untersuchung. zur Physiol. und Pathol. der Nierenfunktion. Arch. f. exp. Pharm. und Pathol. 1906, Bd. LIV, S. 1. 
den Tieren injiziert. Das eine Tier ertrug die Injektionen von 3-6 cg recht gut. Bei dem anderen Tiere, das erheblich mehr Kaliumchromat an aufeinanderfolgenden Tagen (von 0,03-0,15 g) erhielt, trat infolge der Kaliumchromatwirkung der Tod ein. Bei beiden Tieren wurde deutliche Albuminurie und beim zweiten Tier auch Cylindrurie neben kolossalen Beimengungen von Leukocyten zum Urine beobachtet. Die Resultate der Nierenschädigungsversuche sind in Tabelle XII und XIII niedergelegt. In Tabelle XII findet sich zunächst an den ersten Versuchstagen absolut keine Abweichung von der Norm, abgesehen davon, daß unter dem Einfluß der Chromvergiftung die Diurese etwas steigt. Erst am 3. Tage, nach der zweiten Injektion von Kaliumchromat beobachtet man eine etwas gesteigerte Kreatinausscheidung. Am 4. Tage ist dies noch deutlicher. Dabei steigt der Gesamtwert des Kreatinins und Kreatins etwas, während die Stickstoffausscheidung gleich bleibt; der Kreatininwert dagegen bleibt sich fast völlig gleich, ist jedoch niedriger als am ersten Versuchstage. Nach intravenöser Injektion von $0,5 \mathrm{~g}$ Kreatin bei gleichzeitiger subkutaner Injektion von Kaliumchromat findet eine etwas vermehrte Kreatinausscheidung statt, entsprechend den früher erhobenen Befunden nach Kreatininjektionen. Am nächsten Tage (8.) ist die Kreatinausscheidung der Kreatininausscheidung gleich geworden und bleibt auch am 9. Tage fast ebenso groß. Allmählich geht dann der Kreatinwert wieder etwas herunter; unter dem Einfluß von Fleischnahrung kommt sodann eine stark vermehrte Kretinausfuhr zustande trotz fortgesetzter Chromgaben. Nach Auf hören der Fleischfüiterung bleibt trotz starken Rückganges der Gesamtausscheidung von Kreatinin und Kreatin die Kreatinausfuhr hoch. Deutlicher als in Tabelle XII lassen sich in Tabelle XIII die Verhältnisse überblicken. Hier finden wir vom 3. Tage an ein stetices Steigen des Kreatinwertes ohne entsprechende Steigerung des Gesamtwertes für Kreatinin und Kreatin. Am 7. Tage ist die Kreatinmenge der gefundenen Kreatininmenge gleich und am 8. Tage übersteigt sie dieselbe sogar. In der zahlenmäßigen auf Kreatinin berechneten Wiedergabe dieser Verhältnisse kommt der Gegensatz zwischen den normalen Be- 
Tabelle XII.

Hündin $7 \mathrm{~kg}$. Mit Brot und Milch.

\begin{tabular}{|c|c|c|c|c|c|c|}
\hline V.T. & $\begin{array}{c}\text { Urin- } \\
\text { menge } \\
\text { von } \\
6-12 \\
12-6 \\
\text { Uhr } \\
\text { ccm }\end{array}$ & $\mathrm{N}$ & Kreatinin & Kreatin & $\begin{array}{l}\text { Gesamt- } \\
\text { wert für } \\
\text { Kreatinin } \\
\text { und } \\
\text { Kreatin }\end{array}$ & Bemerkungen \\
\hline \multirow[t]{3}{*}{1.} & 80 & 1,09002 & 0,17053 & \multirow{3}{*}{ - } & 0,17053 & \multirow{3}{*}{$\begin{array}{l}\text { Um } 6 \text { Uhr } 0,04 \text { g } \\
\text { Kal. chromat. } \\
\text { subkutan. }\end{array}$} \\
\hline & 25 & 0,30226 & 0,03165 & & 0,03165 & \\
\hline & 105 & 1,39418 & 0,20218 & & 0,20218 & \\
\hline \multirow[t]{3}{*}{2.} & 112 & 1,28315 & 0,08525 & 0,01782 & 0,10307 & \multirow{3}{*}{$\begin{array}{l}\text { Reichl. Alb., keinı } \\
\text { Zylinder. }\end{array}$} \\
\hline & 125 & 0,50966 & 0,03420 & 0,00981 & 0,04401 & \\
\hline & 237 & 1,79281 & 0,11945 & 0,02763 & 0,14708 & \\
\hline \multirow[t]{3}{*}{3.} & 115 & 1,05532 & 0,08469 & 0,00322 & 0,08791 & \multirow{3}{*}{ Urin reichl. Alb. } \\
\hline & 60 & 0,43940 & 0,02700 & 0,02491 & 0,05191 & \\
\hline & 175 & 1,49472 & 0,11169 & 0,02813 & 0,13982 & \\
\hline \multirow[t]{3}{*}{4.} & 150 & 0,79358 & 0,09637 & 0,035570 & 0,13207 & \multirow{3}{*}{$\begin{array}{l}\text { Am 3. Tage } 6 \text { Uhir } \\
\text { abends } 0,03 \mathrm{~g} \\
\text { Kal. Chromat. } \\
\text { subkutan. }\end{array}$} \\
\hline & 100 & 0,555936 & 0,04050 & 0,03314 & 0,07364 & \\
\hline & 250 & 1,35294 & 0,13687 & 0,06884 & 0,20571 & \\
\hline \multirow[t]{3}{*}{5.} & 215 & 1,40637 & 0,11610 & 0,09122 & 0,20732 & \multirow{3}{*}{$\begin{array}{l}2 \% \text { Alb. nach } \\
\text { Eßbach. }\end{array}$} \\
\hline & 35 & 0,48060 & 0,03993 & 0,02753 & 0,06746 & \\
\hline & 250 & 1,88697 & 0,15603 & 0,11875 & 0,27478 & \\
\hline \multirow[t]{3}{*}{6.} & 58 & 0,90748 & 0,06183 & 0,02517 & 0,08700 & \\
\hline & 167 & 1,20265 & 0,09084 & 0,02071 & 0,11155 & \\
\hline & 225 & 2,11013 & 0,15267 & 0,04588 & 0,19855 & \\
\hline \multirow[t]{3}{*}{7.} & 120 & 1,08570 & 0,07710 & 0,01818 & 0,09528 & \multirow{3}{*}{$\begin{array}{l}\text { 1/2\% Alb. Um } 1 \text { Uhır } \\
0,5 \text { g Kreatin in-- } \\
\text { travenös u. 0,04 } \\
\text { Kal. chromat. } \\
\text { subkutan. }\end{array}$} \\
\hline & 124 & 0,65391 & 0,06050 & 0,10150 & 0,16200 & \\
\hline & 244 & 1,73961 & 0,13760 & 0,11968 & 0,25728 & \\
\hline \multirow[t]{3}{*}{8.} & 121 & 1,26735 & 0,09419 & 0,09629 & 0,19048 & \\
\hline & 43 & 0,30452 & 0,02561 & 0,02083 & 0,04644 & \\
\hline & 164 & 1,57187 & 0,11980 & 0,11712 & 0,23692 & \\
\hline
\end{tabular}


Tabelle XII.

Fortsetzung.

\begin{tabular}{|c|c|c|c|c|c|c|}
\hline V. T. & $\begin{array}{c}\text { Urin- } \\
\text { menge } \\
\text { von } \\
6-12 \\
12-6 \\
\text { Uhr } \\
\text { ccm }\end{array}$ & $\mathrm{N}$ & Kreatinin & Kreatin & $\begin{array}{c}\text { Gesamt- } \\
\text { wert für } \\
\text { Kreatinin } \\
\text { und } \\
\text { Kreatin }\end{array}$ & Bemerkungen \\
\hline \multirow[t]{3}{*}{9.} & 177 & 1,54771 & 0,11947 & 0,085334 & 0,20481 & \\
\hline & 45 & 0,47866 & 0,03962 & 0,01244 & 0,05206 & \\
\hline & 222 & 2,02637 & 0,15909 & 0,09778 & 0,25687 & \\
\hline \multirow[t]{3}{*}{10.} & 65 & 0,85528 & 0,06750 & 0,00364 & 0,07114 & \multirow{3}{*}{$\begin{array}{l}\text { Urin: reichl. Alb. } \\
\text { Um } 6 \text { Uhr } 0,05 \mathrm{~g} \\
\text { Kal. chromat. } \\
\text { subkutan. }\end{array}$} \\
\hline & 55 & 0,63474 & 一 & 一 & 0,06187 & \\
\hline & 120 & 1,49002 & 0,06750 & 0,00364 & 0,13301 & \\
\hline \multirow[t]{3}{*}{11.} & 110 & 1,43753 & 0,09900 & 0,02139 & 0,12039 & \multirow{3}{*}{$\begin{array}{l}\text { Fleischnahrung } \\
400 \mathrm{~g} \text { Fleisch. }\end{array}$} \\
\hline & 63 & 1,06356 & 0,04399 & 0,23587 & 0,27986 & \\
\hline & 173 & 2,50109 & 0,14299 & 0,25726 & 0,40025 & \\
\hline \multirow[t]{3}{*}{12.} & 230 & 3,04257 & 0,09624 & 0,17769 & 0,27393 & \multirow{3}{*}{$\begin{array}{l}\text { Fleischnahrung. } \\
200 \mathrm{~g} \text { Fleisch. Um } \\
6 \mathrm{Uhr} 0,06 \mathrm{~g} \mathrm{Kal} . \\
\text { chrom. subkut. }\end{array}$} \\
\hline & 130 & 1,00823 & 0,03900 & 0,11141 & 0,15041 & \\
\hline & 360 & 4,05080 & 0,13524 & 0,28910 & 0,42434 & \\
\hline \multirow[t]{3}{*}{13.} & 175 & 2,37547 & 0,04348 & 0,07271 & 0,11619 & \multirow{3}{*}{ Urin reichl Alb. } \\
\hline & 37 & 0,45714 & 0,03330 & 0,00832 & 0,04162 & \\
\hline & 212 & 2,83261 & 0,07678 & 0,08103 & 0,15781 & \\
\hline
\end{tabular}

ziehungen von Kreatinin zu Kreatin und den hier vorliegenden weniger deutlich zum Ausdruck als in den bei der kolorimetrischen Ablesung gewonnenen, noch nicht umgerechneten Werten. Während man sonst gewohnt ist, nach Invertierung des Kreatins für den Gesantwert Ziffern zu finden, die den für das Kreatinin gefundenen Wert nur um höchstens 2-3 mg übersteigen, beobachtet man hier, daß der Gesamtwert den Kreatininwert um nahezu das Doppelte übersteigt, ohne daß die Gesamtwerte absolut größer werden. Eine Erklärung für diesen Befund liegt nahe. Aus den Autolyseversuchen ist bekannt, daß die Niere aus Kreatin Kreatinin zu bilden im- 


\section{Tabelle XIII.}

Bastardhündin 6600 g. Mit Milch und Brot ernährt.

\begin{tabular}{|c|c|c|c|c|c|c|}
\hline V.T. & $\begin{array}{c}\text { Urin- } \\
\text { menge } \\
\text { von } \\
6-12 ; \\
12-6 \\
\text { Uhr } \\
\text { ccm }\end{array}$ & $\mathrm{N}$ & $\begin{array}{l}\text { Krea- } \\
\text { tinin }\end{array}$ & Kreatin & $\begin{array}{c}\text { Gesamt- } \\
\text { wert für } \\
\text { Krea- } \\
\text { tinin } \\
\text { und } \\
\text { Kreatin }\end{array}$ & Bemerkungen \\
\hline \multirow[t]{3}{*}{1.} & 275 & 3,0950 & 0,08200 & 0,06078 & 0,14278 & \multirow{3}{*}{ Milch und Brot. } \\
\hline & 137 & 0,9219 & 0,03800 & 0,01024 & 0,04824 & \\
\hline & 412 & 4,0169 & 0,12000 & $|0,07102|$ & 0,19102 & \\
\hline \multirow[t]{3}{*}{2.} & 330 & 3,2390 & 0,11622 & 0,03395 & 0,15017 & \multirow{3}{*}{ Milch und Brot. } \\
\hline & 67 & 0,4508 & 0,01605 & - & 0,01605 & \\
\hline & 397 & 3,6898 & 0,13227 & $|0,03395|$ & $|0,16622|$ & \\
\hline \multirow[t]{3}{*}{3.} & 91 & 1,1500 & 0,04188 & 0,00306 & 0,04494 & \multirow{3}{*}{$\begin{array}{l}\text { Milch und Brot. Um } 6 \mathrm{Uhr} \\
0,03 \mathrm{~g} \text { Kal. chromat. }\end{array}$} \\
\hline & 43,5 & 0,3890 & 0,01233 & 0,00122 & 0,01355 & \\
\hline & 134,5 & 1,5390 & 0,05421 & 0,00428 & $|0,05849|$ & \\
\hline \multirow[t]{3}{*}{4.} & 371 & 2,5500 & 0,06768 & 0,00408 & 0,07176 & \multirow{3}{*}{$\begin{array}{l}\text { Um } 6 \text { Uhr } 0,06 \mathrm{~g} \mathrm{Kal.} \mathrm{chro-} \\
\text { mat. }\end{array}$} \\
\hline & 113 & 0,9208 & 0,03935 & 0,00508 & 0,04443 & \\
\hline & 484 & 3,4708 & 0,10703 & 0,00916 & 0,11619 & \\
\hline \multirow[t]{3}{*}{5.} & 505 & 2,5660 & 0,04928 & 0,02148 & 0,07176 & \multirow{3}{*}{$\begin{array}{c}1 / 4-1 / 2 \% \text { Alb. Um } 6 \text { Uhr } \\
0,09 \text { g Kal. chromat. }\end{array}$} \\
\hline & 87 & 0,2785 & 0,00859 & $0,0067 \tilde{s}$ & 0,015:34 & \\
\hline & 592 & 2,8445 & 0,05787 & 0,02923 & 0,08710 & \\
\hline \multirow[t]{3}{*}{6.} & 136 & 0,9906 & 0,05798 & 0,03547 & 0,09345 & \multirow{3}{*}{$\begin{array}{l}\text { Im Urin massenhaft Leuko- } \\
\text { cyten; einzelne Zylinder. } \\
\text { Um } 6 \text { Uhr } 0.15 \mathrm{~g} \mathrm{Kal.chro-} \\
\text { mat. } 6 \% \text { Alb. }\end{array}$} \\
\hline & 11,5 & 0,0958 & - & 二 & - & \\
\hline & 147,5 & 1,0864 & 0,05798 & 0,03547 & $\mid 0,09345$ & \\
\hline 7. & 112 & 0,3915 & 0,00648 & 0,00561 & 0,01209 & $\begin{array}{l}\text { Um } 5 \text { Uhr } 0,15 \mathrm{~g} \text { Kal. chro- } \\
\text { mat. }\end{array}$ \\
\hline 8. & 60 & 0,2275 & 0,00211 & 0,00672 & 0,00883 & $\begin{array}{l}4 \% \text { Alb. nach EBbach. } \\
\text { Exitus. }\end{array}$ \\
\hline
\end{tabular}

stande ist. Wird ihre Funktion gestört, so findet die Kreatininbildung garnicht oder nur unvollkommen statt und es kommt zur vermehrten Kreatinausscheidung. Gegen diese Auffassung lassen sich aber schwerwiegende Bedenken geltend machen. Wenn die Niere tatsächlich Kreatin in Kreatinin umwandelt, warum beobachtet man dann nach Kreatinfütterung oder nach 
Kreatininjektionen niemals Vermehrung des ausgeschiedenen Kreatins? Diese Frage läßt sich nach unseren bisherigen Kenntnissen absolut nicht beantworten; wir wissen nur, daß eingeführtes Kreatin niemals in Kreatinin umgewandelt wird. Ganz einfach liegen also die Verhältnisse nicht und man kann nicht umhin, auch die schon von Voit ${ }^{1}$ ) gemachte Beobachtung, daß nämlich Kreatinin in alkalischer Lösung in Kreatin übergeht, zur Erklärung unserer Befunde heranzuziehen. Nach eingetretener Chromsäurewirkung wird der Harn alkalisch, es ist also möglich, daß dadurch die Kreatinmenge auf Kosten des Kreatinins zunimmt. Eine Entscheidung, welche von beiden Möglichkeiten zutrifft, läßt sich nicht fällen. Mit Rücksicht auf die erwähnten Tatsachen bei der Kreatinfütterung oder -injektion hat jedoch die Annahme, daß es sich bei der Chromnephritis um eine Rückverwandlung von Kreatinin in Kreatin handelt, vieles für sich. Ganz besondere Berücksichtigung scheint mir jedoch der Umstand zu verdienen, daß im Gegensatz zu den Erfahrungen nach Leberschädigung eine quantitative Veränderung des ausgeschiedenen Gesamtwertes für Kreatinin und Kreatin bei der Nierenschädigung nie konstatiert werden konnte.

\section{Zusammenfassung.}

Die Anschauungen über den Kreatinin- und Kreatinstoffwechsel sind bis in die jüngste Zeit noch ungeklärt gewesen. Als treffendes Beispiel hierfür führt af Klercker an, daß selbst in demselben (Noordenschen) Handbuch verschiedene Autoren verschiedene Ansichten über dieselben Fragen äußern und mit Literaturzitaten belegen konnten. Nun sind in letzter Zeit eine ganze Reihe von Untersuchungen erschienen, um die Hauptfragen des Kreatinin- und Kreatinstoffwechsels zu klären. In allen wird die Schwierigkeit der Methodik und die große Menge der Fehlerquellen betont. Es ist daher nicht zu verwundern, wenn erst allmählich völlige Übereinstimmung über die Hauptpunkte des Kreatinin- und Kreatinstoffwechsels erzielt wird. Die beiden Fragen, welche die Ausgangspunkte nahezu der gesamten Untersuchungen darstellen, waren: Wann und wo wird

.) Zeitschr. f. Biol., Bd. IV, S. 93. 
Kreatinin und Kreatin gebildet und - gewissermaßen als Unterfrage dazu - in welcher Beziehung stehen Kreatin und Kreatinin untereinander? Wenn man die älteren Untersuchungsresultate betrachtet, so bekommt man den Eindruck, als ob die beiden Fleischbasen sehr enge physiologische Beziehungen zu einander hätten, als ob Kreatin sehr leicht im Organismus in Kreatinin überginge und umgekehrt. Verstärkt wird dieser Eindruck durch die neueren Befunde von Gottlieb und Stangassinger, die in allen von ihnen untersuchten autolysierten Organen Fermente fanden, welche Kreatin in Kreatinin umwandeln. Die Schwierigkeit in der Deutung ihrer Befunde für den normalen Stoffwechsel ist den genannten Untersuchern, die jeden Schluß aus ihren Autolyseversuchen auf die Stoffwechselvorgänge sorgfältig vermieden, durchaus nicht entgangen, und es erscheint daher zweckmäßig, die Vorgänge bei der Autolyse zunächst für die Ergebnisse von Stoffwechselversuchen unberücksichtigt zu lassen und zu der Beurteilung nur die Fütterungsund Arbeitsversuche heranzuziehen.

Das Kreatinin galt früher als eines der Endprodukte des Eiweißstoffwechsels oder als eine der Vorstufen des Harnstoffs. ${ }^{1}$ ) Die Tatsache einerseits, daß verfüttertes Kreatinin vollständig wieder im Urine erscheint (af Klercker, Hoogenhuyze, Verploegh), daß bei Muskelarbeit die Kreatininausfuhr im Hunger steigt (van Hoogenhuyze, Verploegh), daß das arbeitende Herz Kreatinin oder Kreatin abgibt (Weber) anderseits, lassen das Kreatinin als spezifisches Produkt des Muskelstoffwechsels erscheinen. Nun enthält aber nach Mellanby der Muskel überhaupt kein Kreatinin, sondern nur Kreatin, sodaß der Gedanke naheliegt, daß aus dem Muskelkreatin Kreatinin entstünde. Dieser Annahme stehen jedoch die Fütterungs- und Injektionsversuche von Kreatin direkt entgegen. Es ist kein einziger Fall bekannt, in dem mit Sicherheit im Organismus aus Kreatin Kreatinin entstanden ist. Auf Grund dieser Tatsache und in der Überlegung, daß der Körper nicht aus dem ungiftigen Stoffe einen giftigen bereitet,

1) Bunge, Lehrb. d. Physiol. d. Menschen 1905, S. 149. 
kommt Mellanby zu der von Folin schon vertretenen Ansicht, daß der Kreatinin- und Kreatinstoffwechsel zwei gänzlich verschiedene Vorgänge darstellen. Auf Grund meiner Versuchsergebnisse kann ich dieser Auffassung nur beipflichten; einmal beobachtete auch ich nie Vermehrung des Harnkreatins nach Kreatinfütterung oder nach parenteraler Einfuhr. Dann trat bei der Phosphorvergiftung vermehrte Kreatininausscheidung auf, bei einem Vorgange also, bei dem die Muskulatur so gut wie unbeteiligt war, bei dem jedoch starker Eiweißzerfall vor sich ging. Darnach wäre die Kreatininausscheidung eine Folge des Eiweißzerfalles und der Ort der Kreatininbildung die Leber. Ist deren Funktion gestört, so kommen auch Störungen in der Kreatininausfuhr zustande. Wie aber deckt der Muskel seinen Kreatinbedarf? Hier scheint mir die Fermenttätigkeit einzusetzen. Aus den Autolyseversuchen ist bekannt, daß die Leber, die Niere und die Muskulatur Fermente enthalten, welche Kreatin aus Kreatinin zu bilden imstande sind, und daß diese Fermentvorgänge genau entsprechend dem Bedürfnis des Organismus vor sich gehen, dafür scheint mir ein Beweis zu sein, daß bei Fleischextraktverfütterung, also bei stickstoffreicher Kost, fast alles Kreatin wieder ausgeschieden wird, während Kreatingaben im Hunger so gut wie völlig verschwinden. Die.Hauptfaktoren also, die den Kreatin- und Kreatininstoffwechsel bedingen, sind, abgesehen von dem mit der Nahrung eingeführten Kreatinin und Kreatin, Muskelarbeit und Eiweißumsatz; der Hauptbildungsort für Kreatinin und Kreatin die Leber. Die Regulierung dieser Vorgänge wird durch Fermente besorgt, die entsprechend dem Bedarf des Körpers in Funktion treten.

Die Ergebnisse der vorstehenden Versuche sind kurz zusammengefaßt:

1. Die Kreatinin- und Kreatinausscheidung ist beim gleichmäßig ernährten Tier ziemlich konstant; mit der Nahrung zugeführtes Kreatin oder Kreatinin wird beim gut genährten Tiere alsbald wieder völlig ausgeschieden.

2. Per os oder parenteral zugeführtes Kreatin wird nie in Kreatinin umgewandelt; im Hunger verschwindet parenteral zugeführtes Kreatin so gut wie vollständig. 
3. Leberschädigung und erhöhter Eiweißzerfall haben zunächst Vermehrung der ausgeschiedenen Kreatininmenge, dann Verminderung derselben zur Folge; mit verminderter Kreatininausscheidung geht eine erhöhte Kreatinausfuhr einher; es macht dies die Annahme wahrscheinlich, daß der Ort der Kreatininbildung die Leber sei.

4. Bei der Chromnephritis wird fast alles Kreatinin in Kreatin umgewandelt, wabrscheinlich durch Veränderung der Urinreaktion; quantitative Unterschiede der Gesamtmenge von Kreatinin und Kreatin wurden nicht beobachtet. 